\title{
Reaction-Diffusion Models of Growing Plant Tips: Bifurcations on Hemispheres
}

\author{
Wayne Nagata \\ Department of Mathematics \\ University of British Columbia \\ Vancouver, British Columbia, Canada V6T 1 Z2 \\ nagata@math.ubc.ca \\ Lionel G. Harrison \\ Department of Chemistry \\ University of British Columbia \\ Vancouver, British Columbia, Canada V6T 1Z1 \\ lionel@pepe.chem.ubc.ca \\ Stephan Wehner \\ Department of Mathematics and Statistics \\ Simon Fraser University \\ Burnaby, British Columbia, Canada V5A 1S6 \\ stephan@sugarmotor.net
}

December 20, 2002

\begin{abstract}
We study two chemical models for pattern formation in growing plant tips. For hemisphere radius and parameter values together optimal for spherical surface harmonic patterns of index $l=3$, the Brusselator model gives an $84 \%$ probability of dichotomous branching pattern and $16 \%$ of annular pattern, while the hyperchirality model gives $88 \%$ probability of dichotomous branching and $12 \%$ of annular pattern. The models are two-morphogen reaction-diffusion systems on the surface of a hemispherical shell, with Dirichlet boundary conditions. Bifurcation analysis shows that both models give possible mechanisms for dichotomous branching of the growing tips. Symmetries of the models are used in the analysis.
\end{abstract}




\section{Introduction}

Much of the growth of plants occurs by the elongation of cylindrical stalks or roots by action chiefly at a dome-shaped tip, for which a hemisphere is a reasonable working approximation for mathematical study. Plant development often involves more complex events at the growing tips. Many such events are branching processes which break circular symmetry. Among these, one of the commonest events is dichotomous branching. It is considered to be probably the oldest kind of branching, intrinsic to the evolution of multicellular plants (Zimmermann, 1952). In plant embryos, it generates the pair of seed leaves that characterizes the very large class of flowering plants therefore called dicotyledons. It occurs also in some unicellular plants of large size, that develop complex shapes (Holloway and Harrison, 1999; Dumais and Harrison, 2000). (These seem rather more comparable to plants than to other protists in their development.)

The morphogenetically active region is not a solid hemisphere. In unicellular cases, it is the cell surface (membrane and wall) or possibly a thin layer of cytoskeleton immediately inside the membrane. In multicellular cases, it is a single outermost layer of cells, the tunica, of the growing tip called an apical meristem. For both, a reasonable approximation is a hemispherical shell, the thickness of the active region being much less than its diameter.

No mechanism for the symmetry-breaking at a growing tip is definitely known and accepted. Various mechanisms have been proposed for pattern formation in a thin superficial domain starting with circular symmetry: mechanical buckling (Green et al., 1996); mechanochemical interactions (Goodwin and Trainor, 1985); and chemical reaction-diffusion patterning, following Turing's (1952) postulate that morphological patterns follow corresponding prepatterns in the concentrations of certain chemical substances (morphogens). In Turing's theory, the patterns arise from catalytic and inhibitory reactions of two morphogens, both of which diffuse, but at different rates. Elaborations of this theory, as well as examples, can be found in the books of Nicolis and Prigogine (1977), Meinhardt (1982; 1995), and Harrison (1993). Work of the Brussels and Bordeaux groups relevant to diverse patterns observed in a chlorite-iodide-malonate reaction, a non-biological system known to have Turing dynamics, can be found, e.g. in Verdasca et al. (1992) and Dewel et al. (1995).

In that reaction, diffusivities substantially less than the narrow range in aqueous solutions arise because of the use of a gel and of a large molecule 
in the starch-iodine indicator. In living systems, a wide range of diffusivities is always possible. For instance, in the plant growth we are considering, for unicellular cases some chemical species may be bound to the cell membrane, and for multicellular cases diffusion may be through the narrow cell-to-cell channels known as plasmodesmata. We have used diffusivity ratios of 10 (subsection 3.2) and 12.5 (subsection 3.3). Widely different values of this ratio have been used in published work, ranging at least from 5 [see p. 273 of Harrison (1993)] to 100. The pattern-forming property is usually not very sensitive to this value provided that it is substantially greater than 1, except that very high values tend to give some disorder in patterns of many spots on a plane (Lacalli, 1981; Holloway and Harrison, 1995).

In this paper, we consider the application of reaction-diffusion theory to dichotomous branching at a growing tip. The study arose from a problem encountered in taking computational work from two to three spatial dimensions. In two dimensions, Holloway and Harrison (1999), following Harrison and Kolár (1988), successfully generated repeated dichotomous branching of a loop, by computer modelling of a reaction-diffusion mechanism. Cell outlines were computed matching several diverse shapes in the genus $\mathrm{Mi}$ crasterias of unicellular algae. But if a patterning process on a semicircular loop is intended as a cross-section of a hemispherical shell, the appearance of two concentration maxima on the semicircle is ambiguous. It could correspond to two high-concentration patches on a hemisphere, the pattern to generate dichotomous branching. Or it could represent a section through an annular high-concentration region on the hemisphere. In the latter, circular symmetry has not been broken. But if the conversion of chemical pattern into morphology is that high concentration gives high rate of surface growth, an annular pattern would lead to flattening of the dome shape of the tip. This is sometimes observed in plants, and is another event needing explanation.

A reaction-diffusion pattern is commonly envisaged as arising out of a uniform distribution of the morphogens, that being a homogeneous steady state solution of the dynamic equations. In the present work, we consider that state to be maintained permanently at the boundary of the growing tip, i.e. there is a Dirichlet boundary condition at the equator of the hemisphere. Linearization of Turing-type mechanisms about the homogeneous steady state shows that the early stages of pattern formation should be a simple competition between linearly independent harmonic waveforms appropriate to the geometry to be established according to their eigenvalues, which are exponential growth rates of amplitudes. For the hemisphere, the waveforms are 
spherical surface harmonics $Y_{l, m}$. For any fixed radius of hemisphere and values of kinetic parameters, all $Y_{l, m}$ of the same index $l$ and satisfying the boundary conditions should have the same growth rate. At $l=3$, this means that annular pattern $(m=0)$ and pattern for dichotomous branching (the real linear combinations of $m= \pm 2$ ) should start growing at equal rates. But what happens as the amplitudes grow and linearization is no longer a good approximation?

As a start to three-dimensional modelling of patterning and growth, a study was done (Harrison et al., 2001) comprising a large number of computations (to apparent steady state) for each of three different reaction-diffusion models having substantially different nonlinearities, on a hemispherical shell domain of fixed size, optimal for $l=3$. For all three models, the majority of the final patterns were for dichotomous branching, but a substantial minority (e.g. about $20 \%$ for the Brusselator model) ended with annular pattern, and one model gave another variant also [tabulation, (Harrison et al., 2001)]. These results led to the bifurcation analysis reported here for two of the models. These are the Brusselator (Prigogine and Lefever, 1968) and the hyperchirality system of Harrison and Lacalli (1978). The Brusselator is widely used, and represents a simple, plausible and generic model of reactiondiffusion pattern formation. The hyperchirality system is representative of a different class of models, which possess an additional mathematical symmetry. This symmetry turns out to change some features of the patterns that arise, e.g. in infinite two-dimensional planar surfaces favouring stripes over spots where the Brusselator favours spots. [Computations with radii optimal for $l>3$, reported in (Harrison et al., 2001), showed that, for the hyperchirality model, complex patterns appear that seem to be showing the striping tendency. For $l=2$, but on the complete sphere, see (Hunding and Billing, 1981; Hunding and Brons, 1990).]

In addition to the contrast in mathematical symmetry, the two models exemplify the main contrast in types of chemical mechanism for reactiondiffusion patterning. The Brusellator is an activation-depletion mechanism; the hyperchirality model is an activation-inhibition mechanism [terminology devised by Meinhardt (1982) and used by Harrison (1993)].

The bifurcation analyses reported here have confirmed, at least for dynamic behaviour close to the boundary of the Turing instability, the statistical result of the computations as mentioned above. 


\section{Models}

\subsection{The Brusselator}

In the Brusselator there are two morphogens, one an activator and the other a substance the depletion of which is an effective inhibition, whose concentrations are labelled $X$ and $Y$. These morphogens diffuse, with the depleted substance diffusing faster than the activator. The morphogens represent intermediates in a reaction whose initial reactants have concentrations $A$ and $B$. The rate equations for the morphogens are

$$
\begin{aligned}
& \frac{\partial X}{\partial t}=D_{X} \nabla^{2} X+a A-d X-b B X+c X^{2} Y, \\
& \frac{\partial Y}{\partial t}=D_{Y} \nabla^{2} Y+b B X-c X^{2} Y,
\end{aligned}
$$

in a hemispherical surface $\mathcal{H}_{r}$ of radius $r$, where $\nabla^{2}$ is the Laplacian operator restricted to $\mathcal{H}_{r}, a, b, c, d$ are rate constants, and $D_{X}, D_{Y}$ are diffusion constants with $D_{X}<D_{Y}$. The initial reactants are assumed to be present in sufficiently large quantities throughout the reaction that their concentrations $A$ and $B$ may be considered constants in (1). The chemical concentrations $X, Y, A$ and $B$ are often scaled so that the rate constants are all unity, but we prefer to show explicitly the dependence of the rate equations on the rate constants. This system has a patternless steady solution

$$
X_{0}=\frac{a A}{d}, \quad Y_{0}=\frac{d b B}{c a A}
$$

with uniform morphogen concentrations throughout the hemispherical surface $\mathcal{H}_{r}$. Defining $U, V$ as deviations from the patternless solution, by

$$
X=X_{0}+U, \quad Y=Y_{0}+V,
$$

and expressing (1) in terms of $U$ and $V$ we obtain

$$
\begin{aligned}
& \frac{\partial U}{\partial t}=D_{X} \nabla^{2} U+k_{1} U+k_{2} V+\frac{d b B}{a A} U^{2}+\frac{2 c a A}{d} U V+c U^{2} V, \\
& \frac{\partial V}{\partial t}=D_{Y} \nabla^{2} V+k_{3} U+k_{4} V-\frac{d b B}{a A} U^{2}-\frac{2 c a A}{d} U V-c U^{2} V,
\end{aligned}
$$

in $\mathcal{H}_{r}$, where

$$
k_{1}=b B-d, \quad k_{2}=\frac{c a^{2} A^{2}}{d^{2}}, \quad k_{3}=-b B, \quad k_{4}=-k_{2}=-\frac{c a^{2} A^{2}}{d^{2}} .
$$


On the boundary $\partial \mathcal{H}_{r}$ which is the equator of the hemispherical surface, we assume that the morphogen concentrations $X$ and $Y$ agree with their values for the patternless solution (2), thus the boundary conditions for (4) are the homogeneous Dirichlet conditions

$$
U=0, \quad V=0 \quad \text { on } \quad \partial \mathcal{H}_{r} .
$$

\subsection{The hyperchirality model}

The hyperchirality reaction-diffusion system (Harrison and Lacalli, 1978; Harrison 1993) arose from the fact that biological molecules are usually of a single handedness, but are used to build structures of both handednesses, such as the right and left hands. It was postulated that two handednesses might appear first in such things as two modes of attachment of a supramolecular structure (e.g. protein tetramer) to a membrane, to make "Flatland" enantiomers of a morphogenetic activator substance with concentrations $X_{D}$ and $X_{L}$, interacting with a similarly "hyperchiral" inhibitor substance, with concentrations $Y_{D}$ and $Y_{L}$. However, all that is really required is that there are four morphogens which reduce effectively to two, due to morphogens with the same kinetics grouping into two pairs. This latter requirement is biologically plausible [see the discussions of Harrison (1993) and of Lacalli (1990)]. Like the Brusselator, the hyperchirality system is capable of producing a wide variety of patterns, but the presence of symmetry in the kinetics can affect the type of patterns produced. For example, in an infinite twodimensional plane, Lyons and Harrison (1991) found that the hyperchirality system tends to produce stripes, while the Brusselator tends to produce spots under analogous conditions [discussion of patterns including stripes, spots and honeycomb-like hexagonal arrays can be found in (Dewel et al., 1995)]. The effects of symmetry on pattern selection in the plane is discussed by Golubitsky et al. (1988), where the motivating application is to convection in fluids, but the underlying mathematics is the same for a large class of continuum models including reaction-diffusion sytems.

The rate equations for the morphogens in the hyperchirality system are

$$
\begin{aligned}
\frac{\partial X_{D}}{\partial t}=D_{X} \nabla^{2} X_{D}+k_{X X} A^{2} X_{D}^{2}+k_{X Y} A^{2} Y_{L}^{2} & \\
& \quad-\left(X_{D} A^{2} / P_{X}\right)\left[k_{X X}\left(X_{D}^{2}+X_{L}^{2}\right)+k_{X Y}\left(Y_{D}^{2}+Y_{L}^{2}\right)\right], \\
\frac{\partial X_{L}}{\partial t}= & D_{X} \nabla^{2} X_{L}+k_{X X} A^{2} X_{L}^{2}+k_{X Y} A^{2} Y_{D}^{2}
\end{aligned}
$$




$$
\begin{array}{r}
-\left(X_{L} A^{2} / P_{X}\right)\left[k_{X X}\left(X_{D}^{2}+X_{L}^{2}\right)+k_{X Y}\left(Y_{D}^{2}+Y_{L}^{2}\right)\right] \\
\frac{\partial Y_{D}}{\partial t}=D_{Y} \nabla^{2} Y_{D}+k_{Y X} B^{2} X_{L}^{2}-\left(Y_{D} B^{2} / P_{Y}\right) k_{Y X}\left(X_{D}^{2}+X_{L}^{2}\right) \\
\frac{\partial Y_{L}}{\partial t}=D_{Y} \nabla^{2} Y_{L}+k_{Y X} B^{2} X_{D}^{2}-\left(Y_{L} B^{2} / P_{Y}\right) k_{Y X}\left(X_{D}^{2}+X_{L}^{2}\right),
\end{array}
$$

in $\mathcal{H}_{r}$, where $k_{X X}, k_{X Y}, k_{Y X}$ are rate constants, $D_{X}, D_{Y}$ are diffusion constants, $A, B$ are concentrations of initial reactants, considered constants, and $P_{X}=X_{D}+X_{L}, P_{Y}=Y_{D}+Y_{L}$ are also considered constants. The patternless steady solution corresponds to the racemic mixtures

$$
X_{D}=X_{L}, \quad Y_{D}=Y_{L}
$$

Defining $U$ and $V$ as the differences

$$
U=X_{D}-X_{L}, \quad V=Y_{D}-Y_{L},
$$

and subtracting the second equation in (6) from the first, and the fourth equation from the third, we obtain

$$
\begin{aligned}
& \frac{\partial U}{\partial t}=D_{X} \nabla^{2} U+\left(k_{1 a}+k_{1 b}\right) U+k_{2} V-k_{1 a} U^{3}-k_{5} U V^{2} \\
& \frac{\partial V}{\partial t}=D_{Y} \nabla^{2} V+k_{3} U+k_{4} V-k_{6} U^{2} V
\end{aligned}
$$

in $\mathcal{H}_{r}$, where

$$
\begin{gathered}
k_{1 a}=k_{X X} A^{2} P_{X} / 2, \quad k_{1 b}=-k_{X Y} A^{2} P_{Y}^{2} / 2 P_{X}, \\
k_{2}=-k_{X Y} A^{2} P_{Y}, \quad k_{3}=k_{Y X} B^{2} P_{X}, \quad k_{4}=-k_{Y X} B^{2} P_{X}^{2} / 2 P_{Y}, \\
k_{5}=k_{X Y} A^{2} / 2 P_{X}, \quad k_{6}=k_{Y X} B^{2} / 2 P_{Y} .
\end{gathered}
$$

On the boundary at the equator of the hemispherical surface we take homogeneous Dirichlet conditions

$$
U=0, \quad V=0 \quad \text { on } \quad \partial \mathcal{H}_{r} .
$$




\subsection{Stability and bifurcation}

To study the onset of pattern formation, we begin with a linearized stability analysis of the patternless steady solution $U=0, V=0$ for both models. On the hemispherical surface, it is conventional to take as spatial variables the angles $\theta$ and $\phi$, where $\theta$ is the co-latitude and $\phi$ is the longitude. Then $U$ and $V$ can be expanded in series of the familiar spherical surface harmonics $Y_{l, m}(\theta, \phi)$, where $l=0,1,2, \ldots$ and $m$ is an integer with $|m| \leq l$. Because of the homogeneous Dirichlet boundary conditions (5) and (10), the expansions are restricted to only those surface harmonics that satisfy the boundary condition at the equator: $Y_{l, m}(\pi / 2, \phi)=0$ for all $\phi$. Among all the values of $l$, $l=3$ is the lowest value for which linear combinations of $Y_{l, m}$ give prepatterns for dichotomous branching. In our analysis we choose parameter values so that all $l$ with $l \neq 3$ correspond to stable infinitesimal perturbation modes of the patternless steady state that decay back to the patternless state, while $l=3$ corresponds to slightly unstable perturbation modes that grow away from the patternless state. For the same parameter values in the nonlinear models, we expect patterns to appear that resemble linear combinations of the $Y_{3, m}$. The three surface harmonics for $l=3$ satisfying the homogeneous Dirichlet boundary condition at the equator are

$$
Y_{3,0}(\theta, \phi)=\frac{5}{2} \cos ^{3} \theta-\frac{3}{2} \cos \theta
$$

and

$$
Y_{3, \pm 2}(\theta, \phi)=15 e^{ \pm 2 i \phi} \sin ^{2} \theta \cos \theta .
$$

In order to determine the specific steady patterns that onset in the two models, we reduce both the Brusselator and hyperchirality reaction-diffusion problems to nonlinear bifurcation equations of the form

$$
\begin{aligned}
& \dot{x}=\sigma_{\max } x+B_{1} x^{2}+B_{2} z \bar{z}+C_{1} x^{3}+C_{2} x z \bar{z} \\
& \dot{z}=\sigma_{\max } z+B_{3} x z+C_{3} x^{2} z+C_{4} z^{2} \bar{z} \\
& \dot{\bar{z}}=\sigma_{\max } \bar{z}+B_{3} x \bar{z}+C_{3} x^{2} \bar{z}+C_{4} \bar{z}^{2} z
\end{aligned}
$$

where the real parameter $\sigma_{\max }$ is the common growth rate of the fastest growing $(l=3)$ perturbation modes obtained from the linearized stability analysis of the patternless solution. The variable $x$ is real and represents the amplitude of the $Y_{3,0}$ component of a solution of the reaction-diffusion problem, 
and $z$ is complex and it represents the complex amplitude of the $Y_{3,2}$ component of the solution. Overbars denote complex conjugates, thus the variable $\bar{z}$ represents the complex amplitude of the $Y_{3,-2}$ component of the solution. The coefficients $B_{1}, B_{2}, B_{3}, C_{1}, C_{2}, C_{3}$ and $C_{4}$ are real numbers whose values we must determine. Equilibrium solutions of the bifurcation equations (11) correspond to steady morphogen patterns on the hemispherical surface for the Brusselator or hyperchirality reaction-diffusion problems.

Symmetry plays an important rôle in the analysis of the patterns that form in both models. In a generic system, with no symmetry, only one linearly independent mode would lose stability at a particular set of parameter values. Symmetry is responsible for three linearly independent modes losing stability with the same growth rate $\sigma_{\max }$. Perhaps the most obvious symmetry of both models is the symmetry with respect to rotation about the polar axis of the hemisphere. There is also symmetry with respect to reflections across vertical planes that contain the polar axis. These symmetries are used to simplify the analysis of the bifurcation equations. Another symmetry, whose effects are less obvious, is present in the hyperchirality model. For homogeneous Dirichlet boundary conditions, the fact that (9) has only cubic nonlinearities allows the use of results of Field et al. (1991) on bifurcations on hemispheres. The domain of the problem can be extended mathematically from the hemispherical shell to the spherical shell, and the solutions of the resulting extended problem that are odd with respect to the equator can be found. By restricting such solutions to the upper hemisphere, we recover all solutions to the original problem, but we can exploit the richer symmetry structure of the problem with the spherical shell domain.

Analysis of the bifurcation equations (11) shows that both models produce stable morphogen prepatterns for dichotomous branching. The details of patterns of chemical concentrations differ somewhat between the models, and furthermore the Brusselator model predicts that stable dichotomous branching patterns can occur below the threshold of instability of the patternless solution. In addition, both models allow the possibility of no branching when the patternless solution loses stability. This is because both models have stable annular patterns as well as stable dichotomous branching patterns. In both of the models most initial conditions to lead to dichotomous branching, while a small but significant minority of initial conditions lead to failure to branch (annular pattern). We estimate for both of the models the probabilities that a growing tip will branch dichotomously or fail to branch. 


\section{Bifurcation analysis}

\subsection{Bifurcation equations}

The reduction of time-dependent reaction-diffusion problems to bifurcation equations is standard. Among the various general methods available for reaction-diffusion systems, we mention perturbation expansions using the Fredholm Alternative [e.g. (Nicolis and Prigogine, 1977)], Liapunov-Schimidt reduction [e.g. (Golubitsky and Schaeffer, 1985)] and centre manifold reduction [e.g. (Carr, 1981)]. We prefer centre manifold reduction, because the method has an appealing geometric interpretation, and it preserves symmetry and local dynamics. In particular, the stability of solutions can be determined directly from the bifurcation equations. We do not give details here, but a rigourous justification for the method applied to reaction-diffusion systems can be found in (Carr, 1981).

In this section we discuss the centre manifold reduction of the reactiondiffusion problems (4)-(5) and (9)-(10) to the bifurcation equations (11) governing the dynamics. More technical details of the procedure are given in the Appendix. In each system we suppose that all except one of the parameters are fixed, and consider the remaining parameter as a control parameter, which we denote by $\lambda$. Then the model is written in abstract form as a nonlinear evolution equation

$$
\dot{\boldsymbol{U}}=\boldsymbol{A}(\lambda) \boldsymbol{U}+\boldsymbol{B}(\lambda ; \boldsymbol{U}, \boldsymbol{U})+\boldsymbol{C}(\lambda ; \boldsymbol{U}, \boldsymbol{U}, \boldsymbol{U}),
$$

where the dot denotes differentiation with respect to $t$, the vector function $\boldsymbol{U}=(U(\theta, \phi, t), V(\theta, \phi, t))^{T}$ for each $t \geq 0$ belongs to an infinite-dimensional space of vector functions whose two components each satisfy the homogeneous Dirichlet boundary conditions (5) or (10), and for each $\lambda, \boldsymbol{A}(\lambda)$ is a linear differential operator, sometimes called the Turing operator in the context of reaction-diffusion systems, $\boldsymbol{B}(\lambda ; \cdot, \cdot)$ is a bilinear or quadratic operator, and $\boldsymbol{C}(\lambda ; \cdot, \cdot, \cdot)$ is a trilinear or cubic operator. For the hyperchirality system (9) there are no quadratic terms, thus $\boldsymbol{B}=\mathbf{0}$. This introduces additional symmetry in the hyperchirality system. For both the Brusselator and hyperchirality systems, one solution of the evolution equation (12) is the origin $\boldsymbol{U}=\mathbf{0}$ of the space of vector functions, which corresponds to the patternless steady solution.

The first step of the reduction is to determine the linearized stability of the patternless steady solution, by solving the linear partial differential 
equation eigenvalue problem for the eigenvalues or growth rates $\sigma$,

$$
\boldsymbol{A}(\lambda) \boldsymbol{U}=\sigma \boldsymbol{U}
$$

where $\boldsymbol{U}=(U(\theta, \phi), V(\theta, \phi))^{T}$. This can be accomplished by expanding both components of $\boldsymbol{U}$ in series of the spherical surface harmonic functions $Y_{l, m}(\theta, \phi)$. If all eigenvalues $\sigma$ are real and negative or are complex with negative real parts, then under the linearized dynamics all perturbations of the patternless solution decay exponentially back to $\boldsymbol{U}=\mathbf{0}$, as $t$ increases. In this case it can be shown that $\boldsymbol{U}=\mathbf{0}$ is an asymptotically stable solution of the nonlinear evolution equation (12). If any eigenvalues are positive or have positive real parts, then perturbations that are linear combinations of the corresponding eigenfunctions grow exponentially under the linearized dynamics, and it follows that $\boldsymbol{U}=\mathbf{0}$ is unstable.

In fact, we use parameter values such that all eigenvalues are real, and we denote the largest eigenvalue, or the growth rate of the fastest growing modes, by $\sigma_{\max }$. We find a critical parameter value $\lambda_{0}$ such that, $i$ ) when $\lambda<\lambda_{0}$ all eigenvalues are negative and in particular $\sigma_{\max }<0$, ii) when $\lambda=\lambda_{0}$, there is a zero eigenvalue $\sigma_{\max }=0$ and all the other eigenvalues are negative, and $i i i)$ when $\lambda>\lambda_{0}$ and $\lambda$ is close to $\lambda_{0}$, there is one real positive eigenvalue $\sigma_{\max }>0$ and the remaining eigenvalues all are negative. We interpret this situation as the patternless steady state $\boldsymbol{U}=\mathbf{0}$ losing stability as $\lambda$ increases through $\lambda_{0}$.

For our problems there are three linearly independent vector eigenfunctions, or modes, corresponding to the zero eigenvalue $\sigma_{\max }=0$ for (13) when $\lambda=\lambda_{0}$. We choose the eigenfunctions and label them as

$$
\boldsymbol{U}_{3,0}, \quad \boldsymbol{U}_{3,2}, \quad \boldsymbol{U}_{3,-2},
$$

so that each component of $\boldsymbol{U}_{3, m}$ is a constant multiple of the surface harmonic $Y_{3, m}(\theta, \phi), m=0, \pm 2$. The span of these eigenfunctions is the centre eigenspace

$$
E^{c}=\left\{\boldsymbol{U}^{c}=x \boldsymbol{U}_{3,0}+z \boldsymbol{U}_{3,2}+\bar{z} \boldsymbol{U}_{3,-2} \mid \quad x \in \mathbf{R}, z \in \mathbf{C}\right\},
$$

which is three-dimensional.

According to the centre manifold theorem, if the parameter $\lambda$ is near its critical value $\lambda_{0}$, then the evolution equation (12) has an exponentially attracting, finite-dimensional local centre manifold of solutions $W_{l o c}^{c}$ in the 
infinite-dimensional space of vector functions. This centre manifold $W_{l o c}^{c}$ is tangent to the centre eigenspace $E^{c}$ at the origin of the space of vector functions when $\lambda=\lambda_{0}$, and hence is three-dimensional. Because $W_{l o c}^{c}$ is locally attracting, most transients for small-amplitude solutions of the full system rapidly decay and the local long-term dynamics can be predicted if we know only the dynamics on $W_{l o c}^{c}$, which are governed by a three-dimensional system of first-order ordinary differential equations. In particular, we can predict the existence and stability of bifurcating steady solutions of (12) for $\boldsymbol{U}$ near $\mathbf{0}$, when $\lambda$ is near $\lambda_{0}$. The dynamics on the local centre manifold $W_{l o c}^{c}$ determine which patterns are selected, as $\lambda$ increases through $\lambda_{0}$ and the patternless steady solution loses its stability.

The ordinary differential equations that govern the dynamics on the local centre manifold $W_{l o c}^{c}$ are the bifurcation equations (11), where higher-order terms are neglected. Since the last equation in (11) is just the complex conjugate of the second equation, it is sometimes omitted. In subsections 3.2 and 3.3 we determine the values of the coefficients $B_{1}, B_{2}, B_{3}, C_{1}, C_{2}, C_{3}$, $C_{4}$ in the bifurcation equations (11) for the Brusselator and hyperchirality models, and hence deduce the bifurcation and stability of steady patterned solutions.

It is important to consider the effects of symmetry. Both systems of reaction-diffusion equations together with their hemispherical domains are invariant under rotations about the polar axis of the hemisphere

$$
(\theta, \phi) \mapsto(\theta, \phi+\tau)
$$

for any angle $\tau$, and under the reflection

$$
(\theta, \phi) \mapsto(\theta,-\phi)
$$

Under rotations (15), the critical eigenfunctions transform as

$$
\boldsymbol{U}_{3,0} \mapsto \boldsymbol{U}_{3,0}, \quad \boldsymbol{U}_{3,2} \mapsto e^{i 2 \tau} \boldsymbol{U}_{3,2}, \quad \boldsymbol{U}_{3,-2} \mapsto e^{-i 2 \tau} \boldsymbol{U}_{3,-2}
$$

while under the reflection (16) they transform as

$$
\boldsymbol{U}_{3,0} \mapsto \boldsymbol{U}_{3,0}, \quad \boldsymbol{U}_{3,2} \mapsto \boldsymbol{U}_{3,-2}, \quad \boldsymbol{U}_{3,-2} \mapsto \boldsymbol{U}_{3,2}
$$

Therefore in the centre eigenspace (14) with coordinates $(x, z, \bar{z})$ the rotations (15) are represented by

$$
(x, z, \bar{z}) \mapsto\left(x, e^{i 2 \tau} z, e^{-i 2 \tau} \bar{z}\right),
$$


and the reflection (16) is represented by

$$
(x, z, \bar{z}) \mapsto(x, \bar{z}, z) .
$$

It is a well-known result in centre manifold theory (Ruelle 1973) that such symmetries carry through the centre manifold reduction, and therefore (11) is equivariant under (17) and (18). In fact, the equivariance has already been used to simplify the form of (11); otherwise many more terms (all of which must vanish, due to the symmetries) would appear.

The equivariance of the bifurcation equations (11) under the symmetries (17) and (18) has consequences which help to determine the dynamics. If we have a solution of (11), then transforming the solution by a rotation (17) or reflection (18) gives another solution of (11). It then follows that the $x$-axis is invariant for (11): solutions starting at $t=0$ in the set $\{z=0\}$ remain there for all $t$. Solutions on the $x$-axis correspond to solutions of the reactiondiffusion system with circular symmetry, such as annular patterns. Another consequence is that if there is an equilibrium solution $S$ of (11) with $z \neq 0$, then it is not isolated, but must belong to a continuous circle of equilibrium solutions, each obtained by a rotation (17) of $S$. Such equilibrium solutions of (11) with $z \neq 0$ correspond to patterns in the reaction-diffusion system that do not have circular symmetry, such as dichotomous branching patterns. These patterns must occur in continuous families, since each pattern can be rotated any amount about the polar axis by (15) to produce another pattern in the same family.

\subsection{Pattern formation in the Brusselator model}

In this subsection we give pattern formation results for the Brusselator model (4)-(5). We first describe the linearized stability analysis of the patternless steady state, and then the results of the centre manifold reduction to bifurcation equations. The bifurcation equations are analyzed, and the results are interpreted in terms of patterns for the Brusselator model. The analysis described here leads to basins of attraction indicating that, starting from random noise about the patternless steady state, patterning events should give dichotomous branching with $84 \%$ probability and annular pattern with $16 \%$ probability. Numerical solution of the dynamics (Harrison et al., 2001) further from the Turing instability boundary gave, in a set of 103 computations, $82 \%$ branched and $18 \%$ annular. 
In the Brusselator equations (4), we fix all parameters except the product $b B$ of the rate constant $b$ and the chemical concentration $B$. Then we write (4)-(5) in the abstract form (12), taking $\lambda=b B$, and the operators $\boldsymbol{A}, \boldsymbol{B}$, $\boldsymbol{C}$ given by

$$
\begin{aligned}
\boldsymbol{A}(\lambda) \boldsymbol{U} & =\left(\begin{array}{c}
\left(D_{X} \nabla^{2}+k_{1}\right) U+k_{2} V \\
k_{3} U+\left(D_{Y} \nabla^{2}+k_{4}\right) V
\end{array}\right) \\
\boldsymbol{B}\left(\lambda ; \boldsymbol{U}_{1}, \boldsymbol{U}_{2}\right) & =\left(\begin{array}{c}
\frac{d b B}{a A} U_{1} U_{2}+\frac{c a A}{d}\left(U_{1} V_{2}+U_{2} V_{1}\right) \\
-\frac{d b B}{a A} U_{1} U_{2}-\frac{c a A}{d}\left(U_{1} V_{2}+U_{2} V_{1}\right)
\end{array}\right), \\
\boldsymbol{C}\left(\lambda ; \boldsymbol{U}_{1}, \boldsymbol{U}_{2}, \boldsymbol{U}_{3}\right) & =\left(\begin{array}{c}
\frac{1}{3} c\left(U_{1} U_{2} V_{3}+U_{1} U_{3} V_{2}+U_{3} U_{2} V_{1}\right) \\
-\frac{1}{3} c\left(U_{1} U_{2} V_{3}+U_{1} U_{3} V_{2}+U_{3} U_{2} V_{1}\right)
\end{array}\right) .
\end{aligned}
$$

The operators $\boldsymbol{B}(\lambda ; \cdot, \cdot)$ and $\boldsymbol{C}(\lambda ; \cdot, \cdot, \cdot)$ are defined to be symmetric in their arguments, and $\boldsymbol{C}$ is in fact independent of $\lambda$.

The eigenvalue problem (13) for the linearization of (12) about the patternless steady solution becomes

$$
\begin{gathered}
D_{X} \nabla^{2} U+k_{1} U+k_{2} V=\sigma U, \\
D_{Y} \nabla^{2} V+k_{3} U+k_{4} V=\sigma V, \\
U(\pi / 2, \phi)=0, \quad V(\pi / 2, \phi)=0 .
\end{gathered}
$$

We expand $U$ and $V$ in terms of spherical surface harmonics

$$
U(\theta, \phi)=\sum_{l, m} u_{l, m} Y_{l, m}(\theta, \phi), \quad V(\theta, \phi)=\sum_{l, m} v_{l, m} Y_{l, m}(\theta, \phi),
$$

where the sums are taken over only those integers $l=0,1,2, \ldots,-l \leq m \leq l$ such that the homogeneous Dirichlet boundary condition $Y_{l, m}(\pi / 2, \phi)=0$ is satisfied. Substituting the expansions (23) into the eigenvalue problem (22), we obtain a sequence of algebraic eigenvalue problems for each $l, m$ :

$$
\left(\begin{array}{cc}
-\omega_{l}^{2} D_{X}+k_{1} & k_{2} \\
k_{3} & -\omega_{l}^{2} D_{Y}+k_{4}
\end{array}\right)\left(\begin{array}{c}
u_{l, m} \\
v_{l, m}
\end{array}\right)=\sigma\left(\begin{array}{c}
u_{l, m} \\
v_{l, m}
\end{array}\right),
$$

where $\omega_{l}^{2}=l(l+1) / r^{2}$.

The eigenvalues $\sigma$ depend only on $l$ and are given by

$$
2 \sigma=k_{1}+k_{4}-\omega_{l}^{2}\left(D_{X}+D_{Y}\right) \pm\left(\beta^{2}+4 k_{2} k_{3}\right)^{1 / 2},
$$


where $\beta=k_{4}-k_{1}-\omega_{l}^{2}\left(D_{Y}-D_{X}\right)$. We use parameter values so that $\beta^{2}+$ $4 k_{2} k_{3}>0$ for all $l$, and hence all the eigenvalues are real. Then we find specific conditions for which the largest eigenvalue $\sigma_{\max }$ is zero. To do so, and we maximize $\sigma$ with respect to $\omega_{l}^{2}$, and find that the maximum value of $\sigma$ is

$$
\sigma_{\max }=\left[(1+\delta) k_{1}+(1-\delta) k_{4}+\beta \delta-(\beta / \delta)\right] / 2,
$$

where $\delta=\left(D_{X}+D_{Y}\right) /\left(D_{Y}-D_{X}\right)$. This maximum value is attained when

$$
\beta=-\left(\frac{4 \delta^{2} k_{2} k_{3}}{1-\delta^{2}}\right)^{1 / 2}, \quad \omega_{l}^{2}=\frac{k_{4}-k_{1}-\beta}{D_{Y}-D_{X}} .
$$

If all parameters are fixed except for the product $b B$, the expression for $\sigma_{\max }$ can be considered a smooth function of $b B$.

Parameter values are chosen so that the most unstable modes could correspond to dichotomous branching. We set

$$
\begin{gathered}
D_{X}=10^{-5}, \quad D_{Y}=10^{-4}, \quad A=1, \\
a=0.045, \quad c=1.8, \quad d=0.05,
\end{gathered}
$$

and then numerically solve the nonlinear equation $\sigma_{\max }(b B)=0$ for $b B$, to find

$$
b B=0.367,
$$

with

$$
\omega_{l}^{2}=8540 \text {. }
$$

Thus, for parameter values (25)-(27), all the eigenvalues are real, and the largest eigenvalue $\sigma_{\max }$ is zero. All the other eigenvalues $\sigma$ are strictly negative, and uniformly bounded away from zero.

The radius $r$ of the hemispherical domain is selected so that the zero eigenvalue $\sigma_{\max }=0$ corresponds to modes whose components are proportional to the surface harmonics $Y_{3,0}(\theta, \phi)$ and $Y_{3, \pm 2}(\theta, \phi)$. For $l=3$, the value of $r$ corresponding to the critical value of $\omega_{l}^{2}$ given by (28) is

$$
r_{0}=0.0375
$$

We summarize the results of the linearized stability analysis: if all parameters except $b B$ are fixed at the values given by (25)-(26), and if $r=r_{0}$, then for $b B$ less than the critical value given by (27), we have $\sigma_{\max }<0$ and hence 
all eigenvalues of the eigenvalue problem (22) are negative. This implies that the patternless steady solution $U=0, V=0$ of (4)-(5) is asymptotically stable. If $b B$ increases past the critical value, then $\sigma_{\max }$ increases past zero, and three linearly independent modes all become unstable together. For $b B$ at the critical value, there is a bifurcation of steady solutions of the nonlinear problem (4)-(5) and the analysis of the bifurcation will reveal which pattern is selected. If $r$ is not exactly equal to $r_{0}$, but is close to it, then the same bifurcation occurs, but at a slightly different critical value of $b B$.

Following the steps outlined in Section 2 and the Appendix, we reduce the Brusselator model to the bifurcation equations (11). For $r=r_{0}$ and the parameter values (25)-(27), we obtain numerical values for the coefficients in (11):

$$
\begin{gathered}
B_{1}=-0.00498, \quad B_{2}=B_{3}=-5 B_{1}=0.0249 \\
C_{1}=-0.00605, \quad C_{2}=-0.0956, \quad C_{3}=-0.356, \quad C_{4}=-0.0681 .
\end{gathered}
$$

For more details of the computations, see the Appendix.

We now gather information on the branches of equilibrium solutions associated with the bifurcation at the critical value of $b B$ characterized by $\sigma_{\max }=0$. To find equilibrium solutions of (11) with $z \neq 0$, we solve

$$
\begin{aligned}
& 0=\sigma_{\text {max }} x+B_{1} x^{2}+B_{2}|z|^{2}+C_{1} x^{3}+C_{2} x|z|^{2}, \\
& 0=\sigma_{\text {max }}+B_{3} x+C_{3} x^{2}+C_{4}|z|^{2} .
\end{aligned}
$$

There is a transcritical bifurcation at $\sigma_{\max }=0, x=0,|z|=0$. We use the second equation in (32) to eliminate $|z|^{2}$ and find a saddle-node bifurcation at $\sigma_{\max }=\sigma_{1}=-0.00131, x=0.105,|z|=0.115$. Solutions on the branches given by (32) correspond to steady patterns for the Brusselator model that are not rotationally symmetric. These are circles of solutions with the same value of $|z|$, corresponding to dichotomous branching patterns for the Brusselator that are related by the rotations $(\theta, \phi) \mapsto(\theta, \phi+\tau)$. We label these solutions as $D B+$ and $D B-$. The Brusselator dichotomous branching patterns are approximated at leading order by $\boldsymbol{U}$ whose components are specific linear combinations of the surface harmonics $Y_{3,0}(\theta, \phi), Y_{3,2}(\theta, \phi)$ and $Y_{3,-2}(\theta, \phi)$. A typical dichotomous branching pattern is illustrated in Figure 1.

On the $x$-axis, an invariant subspace, the dynamics are given by $z=0$ and

$$
\dot{x}=\sigma_{\max } x+B_{1} x^{2}+C_{1} x^{3} .
$$




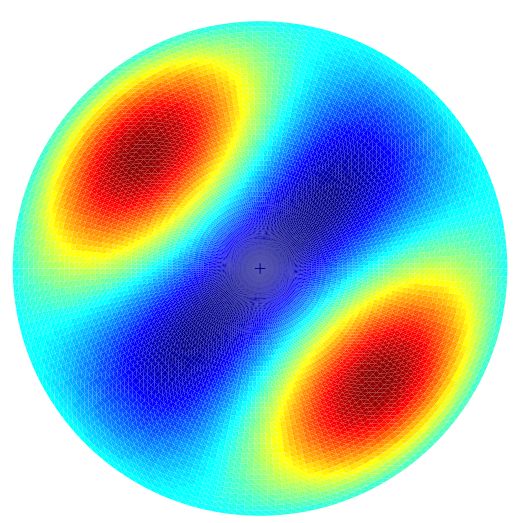

Figure 1: Plan view (i.e. projection of the hemispherical shell onto its equatorial plane) of a dichotomous branching pattern for the Brusselator model, colour-coded for concentration of the morphogen $X$ (dark red, highest; dark blue, lowest) as described in Harrison et al. (2001). This pattern corresponds approximately to a linear combination of $Y_{3,0}$ and $Y_{3,2}$ with a slightly larger coefficient for the latter. Branching will occur if $X$ catalyzes growth of the initially hemispherical surface. 


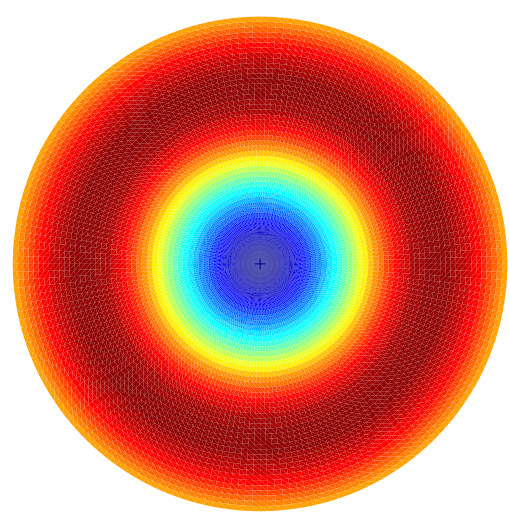

Figure 2: An annular pattern, colour-coded as Figure 1. This pattern is quite close to $Y_{3,0}$. $X$-catalyzed growth would lead to a cup-shaped or crater-like tip, which is sometimes observed as an aberrant form in plant growth where the normal development is branching.

The origin $x=0$ is always an equilibrium solution of (33), corresponding to the patternless steady solution of the Brusselator model. Nonzero equilibrium solutions are given by

$$
0=\sigma_{\max }+B_{1} x+C_{1} x^{2}
$$

There is a transcritical bifurcation at $\sigma_{\max }=0, x=0, z=0$, and we locate a saddle-node bifurcation at $\sigma_{\max }=\sigma_{2}=-0.00102, x=-B_{1} /\left(2 C_{1}\right)=$ $-0.412, z=0$. Solutions on the branches given by (34), labelled $A+$ and $A-$, correspond to steady annular patterns with circular symmetry in the Brusselator model. To leading order, the patterns are approximated by vector functions $\boldsymbol{U}$ whose components are both proportional to the surface harmonic $Y_{3,0}(\theta, \phi)$. See Figure 2 .

The stability of solutions on the branches are determined by linearizing the reduced equations (11) about the equilibrium solutions. The results are summarized in the bifurcation diagram Figure 3, where the amplitudes of the equilibrium solutions are plotted against the values of the parameter $\sigma_{\max }$. The stability of the equilibrium solutions on the branches are indicated. On the branches of dichotomous branching solutions $D B+$ and $D B-$, the linearizations about the equilibria each have a zero eigenvalue, corresponding 


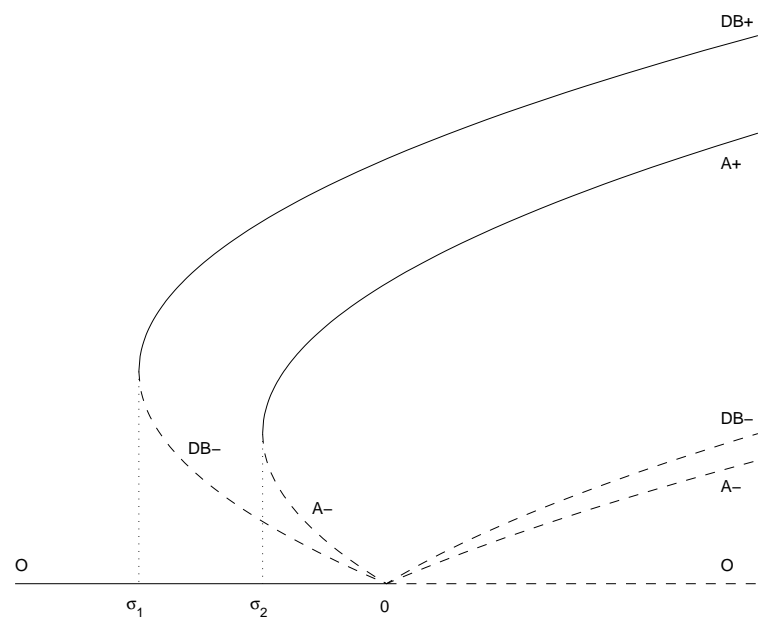

Figure 3: Schematic bifurcation diagram for the Brusselator model. Values of $\sigma_{\max }$ are plotted along the horizontal axis, and the amplitudes of the equilibrium solutions are plotted along the vertical axis. Solid lines represent branches of stable solutions, and dashed lines represent branches of unstable solutions. $O$ indicates the patternless solution, $D B+$ and $D B$ - indicate circles of dichotomous branching pattern solutions (Figure 1), $A+$ and $A-$ indicate annular pattern solutions (Figure 2). 


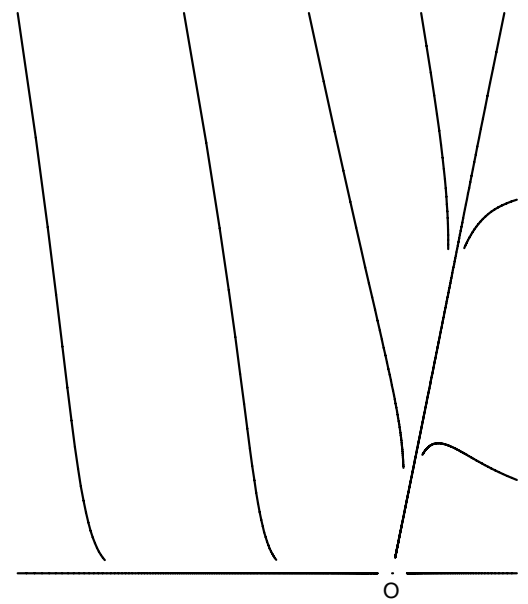

Figure 4: Phase portrait of the bifurcation equations for Brusselator model when $\sigma_{\max }<\sigma_{1}$. The patternless solution $O$ is stable. In this and subsequent two-dimensional phase portraits, the horizontal direction represents the $x$-coordinate, while the vertical direction represents the amplitude $|z|$ of the $z$-coordinate, and the origin is the point $O$. The three-dimensional phase portraits for (11) are generated by rotating the two-dimensional phase portraits about the horizontal axis.

the fact that perturbations of the solutions that are simply rotations along the circles of solutions (translations along group orbits) $(\theta, \phi) \mapsto(\theta, \phi+\tau)$ neither grow nor decay. The growth or decay of perturbations in directions transverse to the rotations or group orbits determine the stability of the dichotomous branching solutions.

For $\sigma_{\max }<\sigma_{1}$ the local phase portrait of (11) has a single, asymptotically stable (or attracting), equilibrium solution $O$ at the origin. See Figure 4. For the Brusselator model, locally only the patternless steady solution $\boldsymbol{U}=\mathbf{0}$ exists when $\sigma_{\max }<\sigma_{1}$, and it is stable.

For $\sigma_{1}<\sigma_{\max }<\sigma_{2}$ the origin $O$ is still a stable equilibrium solution, but also there are two circles of equilibrium solutions $D B+$ and $D B-$ for (11) with constant $|z|$. In the directions normal to these circles, the $D B+$ solutions are attracting while the $D B$ - solutions have a saddle structure, hence are unstable. See Figure 5. This situation corresponds in the Brusselator to the existence of two families of dichotomous branching patterned solu- 


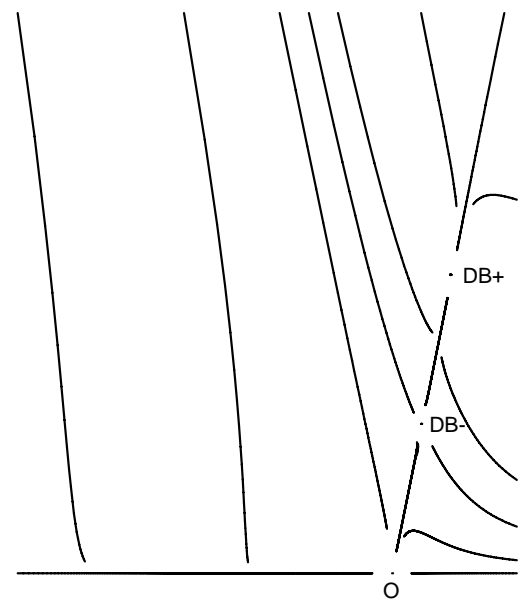

Figure 5: Phase portrait of the bifurcation equations for the Brusselator model when $\sigma_{1}<\sigma_{\max }<\sigma_{2}$. The patternless solution $O$ and dichotomous branching pattern solutions $D B+$ are stable. The dichotomous branching pattern solutions $D B-$ are unstable.

tions, one of which $(D B+)$ consists of stable solutions and the other $(D B-)$ consists of unstable solutions.

When $\sigma_{2}<\sigma_{\max }<0$, the reduced system (11) has a stable equilibrium solution $O$ at the origin, the two circles of equilibrium solutions $D B+$ and $D B$ - described previously, and in addition a pair of equilibrium solutions on the $x$-axis, one $(A+)$ stable and the other $(A-)$ unstable. See Figure 6 . The solutions $A+$ and $A$ - on the $x$-axis for (11) correspond to steady annular patterned solutions for the Brusselator system.

The same types of patterned solutions exist for $\sigma_{\max }>0$, except that their relative positions in phase space change from the previous case, and the origin $O$ is now unstable. See Figure 7.

From the bifurcation analysis, we see that for the Brusselator model, there are stable dichotomous branching solutions even for parameter values for which the patternless solution is still stable. For $\sigma_{\max }>\sigma_{1}$ there are multiple attractors, and which pattern is selected depends on the initial condition. However, if we take a random distribution of initial conditions clustered in a small enough neighbourhood of the patternless solution $O$, for $\sigma_{\max }<0$ most solutions will decay to the patternless solution as $t \rightarrow \infty$. In contrast, for 


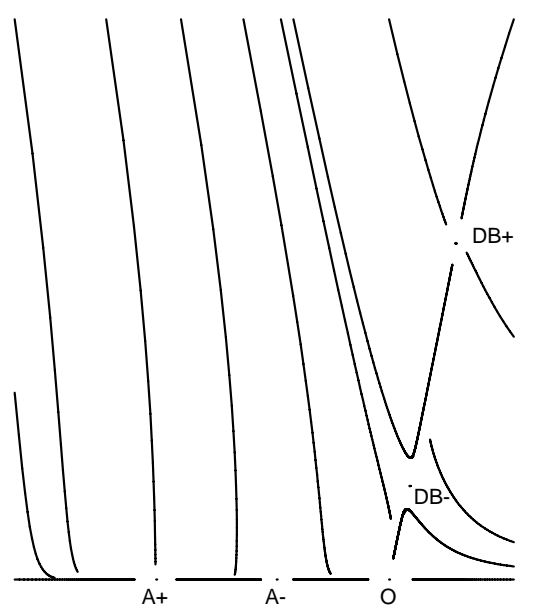

Figure 6: Phase portrait of the bifurcation equations for the Brusselator model when $\sigma_{2}<\sigma_{\max }<0$. The patternless solution $O$, annular pattern solution $A+$ and dichotomous branching pattern solutions $D B+$ are stable. The annular pattern solution $A$ - and dichotomous branching pattern solutions $D B$ - are unstable. 


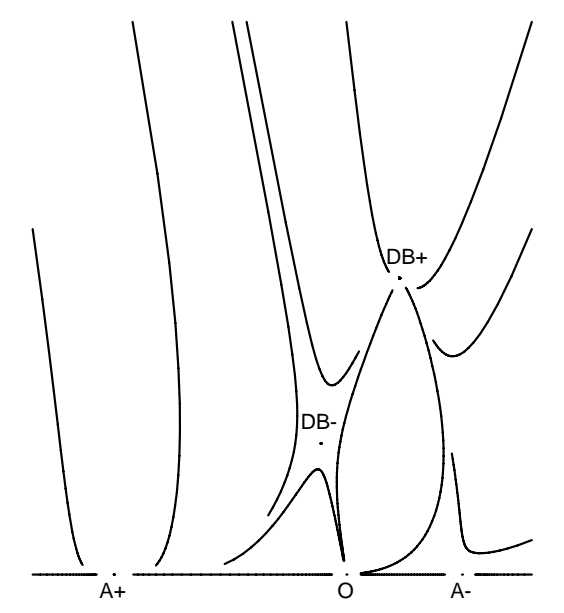

Figure 7: Phase portrait of the bifurcation equations for the Brusselator model when $\sigma_{\max }>0$. The annular pattern solution $A+$ and dichotomous branching pattern solutions $D B+$ are stable. The patternless solution $O$, the annular pattern solution $A$ - and the dichotomous branching pattern solutions $D B$ - are unstable. 
$\sigma_{\max }>0$ solutions that start at $t=0$ near the patternless solution approach one of the dichotomous branching or annular patterned solutions as $t \rightarrow \infty$. The amplitudes of these solutions are already well developed at the critical parameter values for which the patternless solution loses its stability.

For $\sigma_{\max }>0$, we estimate the probability that a randomly selected initial condition near the patternless state will lead to one pattern or another. In Figure 7 we see that the basin boundary between the $D B+$ and $A+$ solutions is determined by the positions of the unstable $D B$ - solutions. The location of the $D B$ - solutions can be approximated from the truncated equations

$$
\begin{aligned}
& 0=\sigma_{\max } x+B_{1} x^{2}+B_{2}|z|^{2}, \\
& 0=\sigma_{\max }+B_{3} x,
\end{aligned}
$$

from which we obtain

$$
x=-\sigma_{\max } / B_{3}, \quad|z|=\sigma_{\max }\left(B_{3}-B_{1}\right)^{1 / 2} /\left(B_{3}^{2} B_{2}\right)^{1 / 2} .
$$

Hence the solid cone $C$ in the three-dimensional $(x, z)$ space with vertex the origin $O$ and side containing the circle of unstable equilibrium solutions $D B$ - has a cross-section that makes the angle $\alpha$ with the $x$-axis at the origin, where $\tan \alpha=\left(B_{3}-B_{1}\right)^{1 / 2} / B_{2}^{1 / 2}=(6 / 5)^{1 / 2}$. We approximate the basin of attraction of the equilibrium point $A+$ by the interior of $C$. The interior of the cone $C$ intersected with the interior of a sphere centred at the origin $O$ occupies a relative volume of $(1-\cos \alpha) / 2=0.16$ with respect to the sphere. Thus we estimate that if initial conditions are chosen at random near the patternless solution for the Brusselator system, approximately $16 \%$ of them will generate solutions that approach the stable annular patterned solution as $t \rightarrow \infty$, while the remaining $84 \%$ will approach one of the stable dichotomous branching patterned solutions. Since, in equation (61) of the Appendix, the coefficients $B_{1}, B_{2}$ and $B_{3}$ are all proportional to the same parameter-dependent expression, the angle $\alpha$ for the basin of attraction is independent of parameters.

\subsection{Pattern formation in the hyperchirality model}

Now we consider the onset of patterns in the hyperchirality model (9)-(10). The reduction to bifurcation equations is similar to that for the Brusselator model, but simpler due to the lack of quadratic nonlinear terms. The symmetry in the hyperchirality model that is not present in the Brusselator 
model can be exploited to give additional information about the patterns that appear. The analysis indicates that patterning events should give dichotomous branching with $88 \%$ probability and annular pattern with $12 \%$. Numerical solution of the dynamics (Harrison et al., 2001) gave, in a set of 415 computations, three types of pattern: branching $73 \%$, annular 14\%, and a pattern described as "keyhole" $13 \%$.

In (9) we consider all parameters as fixed, except the rate constant $k_{X X}$, which we treat as a control parameter. We write (9)-(10) in the abstract form (12) with $\lambda=k_{X X}$, and

$$
\begin{aligned}
\boldsymbol{A}(\lambda) \boldsymbol{U} & =\left(\begin{array}{c}
\left(D_{X} \nabla^{2}+k_{1 a}+k_{1 b}\right) U+k_{2} V \\
k_{3} U+\left(D_{Y} \nabla^{2}+k_{4}\right) V
\end{array}\right) \\
\boldsymbol{B}\left(\lambda ; \boldsymbol{U}_{1}, \boldsymbol{U}_{2}\right) & =\left(\begin{array}{c}
0 \\
0
\end{array}\right), \\
\boldsymbol{C}\left(\lambda ; \boldsymbol{U}_{1}, \boldsymbol{U}_{2}, \boldsymbol{U}_{3}\right) & =\left(\begin{array}{c}
-k_{1 a} U_{1} U_{2} U_{3}-\frac{1}{3} k_{5}\left(U_{1} V_{2} V_{3}+U_{2} V_{1} V_{3}+U_{3} V_{1} V_{2}\right) \\
-\frac{1}{3} k_{6}\left(U_{1} U_{2} V_{3}+U_{1} U_{3} V_{2}+U_{2} U_{3} V_{1}\right)
\end{array}\right.
\end{aligned}
$$

The eigenvalue problem (13) to determine the linearized stability of the patternless state $\boldsymbol{U}=\mathbf{0}$ in the hyperchirality model takes the same form (22) as for the Brusselator if we put $k_{1}=k_{1 a}+k_{1 b}$, and the analysis is very similar. Fixing parameter values

$$
\begin{gathered}
D_{X}=4.4 \times 10^{-5}, \quad D_{Y}=5.5 \times 10^{-4}, \quad A=1, \quad B=1, \\
P_{X}=1, \quad P_{Y}=1, \quad k_{X Y}=0.2, \quad k_{Y X}=1.1,
\end{gathered}
$$

we solve $\sigma_{\max }=0$ to find

$$
k_{X X}=0.643 .
$$

If the radius $r$ of the hemisphere $\mathcal{H}_{r}$ is fixed at

$$
r_{0}=0.0772,
$$

then the modes that correspond to the zero eigenvalue $\sigma_{\max }=0$ have components that are proportional to $Y_{l, m}(\theta, \phi)$ with $l=3$ and $m=0, \pm 2$. Thus, if $r=r_{0}$ and all parameter values except $k_{X X}$ are fixed according to (39) and (40), and if $k_{X X}$ is increased through the critical value (41), then the largest eigenvalue $\sigma_{\max }$ increases through zero, as three linearly independent modes all lose stability. 
The fact that there are only cubic nonlinear reaction terms in the hyperchirality model allows us to take advantage of the resulting symmetry, and obtain some useful information about the solutions. Since $\boldsymbol{B}=\mathbf{0}$, the hyperchirality system written as (12) is odd under the operation

$$
\boldsymbol{U} \rightarrow-\boldsymbol{U}
$$

Because of this additional symmetry, we can use results of Field et al. (1991) on bifurcation on hemispheres: we find solutions of (12) on the surface of the hemisphere $\mathcal{H}_{r_{0}}$ of radius $r_{0}$, with homogeneous Dirichlet boundary conditions, by first extending the domain of the problem to the surface of the sphere $\mathcal{S}_{r_{0}}$ with the same radius, and then seeking solutions of the extended problem on $\mathcal{S}_{r_{0}}$ that are odd about the equator. The restrictions of such solutions to the upper hemisphere $\mathcal{H}_{r_{0}}$ recover all solutions of the original Dirichlet boundary value problem. The bifurcation problem on the spherical domain $\mathcal{S}_{r_{0}}$ is symmetric with repect to the group of orthogonal $3 \times 3$ matrices, and the generic bifurcations of steady solutions in the presence of O(3)-symmetry have been classified by Chossat et al. (1990).

We therefore extend the spatial domain of our problem by considering the bifurcation of steady solutions for the hyperchirality model defined on the surface of the sphere $\mathcal{S}_{r_{0}}$ of radius $r_{0}$, so that the new bifurcation problem has $\mathbf{O}(3)$-symmetry. For the case $l=3$, generically there are precisely three branches of steady solutions that bifurcate when the patternless solution loses stability. The solution branches are characterized by the symmetries (conjugacy classes of isotropy subgroups) possessed by the solutions. One branch of steady solutions has isotropy subgroup (conjugate to) $\mathbf{O}(2)^{-}$, and these solutions are invariant under all rotations about an axis through the origin, and are odd under reflections through the plane perpendicular to the axis of rotational symmetry. Another branch of steady solutions has isotropy subgroup $\mathrm{O}^{-}$; these solutions have a kind of octahedral symmetry, invariant under rotations of $\pi / 2$ radians about any of three mutually orthogonal axes through the origin composed with a reflection. On the remaining branch of steady solutions are sector solutions with isotropy subgroup $\mathbf{D}_{6}^{d}$, that are invariant under rotations of $\pi / 3$ about an axis of symmetry composed with a reflection. For the sector solutions there are three nodal circles on the surface of the sphere that intersect the axis of symmetry. The branches of steady solutions described in this paragraph consist of group orbits of solutions families of solutions that can be transformed into one another by the action of any matrix in $\mathbf{O}(3)$, such as rotations about an axis of symmetry, or 
reflections across a plane of symmetry. Corresponding to each of the three isotropy subgroups are fixed-point subspaces, which are finite-dimensional subspaces that are invariant under the time evolution of the hyperchirality system on the surface of the sphere. For each steady solution on a branch, the fixed-point subspace is one-dimensional, however, each of the bifurcating steady solutions belongs to a continuous family of bifurcating steady solutions on the same group orbit of solutions.

Following Field et al. (1991) we look for the bifurcating steady solutions on the surface of the sphere $\mathcal{S}_{r_{0}}$ that are odd about reflections through the equatorial mid-plane. For solutions on the branch with isotropy subgroup $\mathbf{O}(2)^{-}$, there is a pair of solutions that are odd about the equator: a solution that is invariant under rotations about the polar axis, and its negative. The restrictions of these two solutions to the upper hemisphere $\mathcal{H}_{r_{0}}$ are a pair of annular patterns similar to those for the Brusselator model (Figure 2). Among the solutions with isotropy subgroup $\mathrm{O}^{-}$, those that are odd about the equator are invariant under rotations of $\pi / 2$ radians about the polar axis composed with a reflection. The restrictions of these solutions to $\mathcal{H}_{r_{0}}$ comprise a family of dichotomous branching patterns that can be transformed into one another by rotations about the polar axis. See Figure 8. Sector solutions with isotropy subgroup $\mathbf{D}_{6}^{d}$ are odd about the equator if the axis of symmetry lies in the equatorial mid-plane and one of the three nodal circles coincides with the equator. Restricting these solutions to the upper hemisphere $\mathcal{H}_{r_{0}}$ gives a family of sector patterns that are transformed into each other by rotations about the polar axis. See Figure 9 . Since the subset $\mathcal{U}$ of all solutions of the hyperchirality system, defined on the surface of the entire sphere $\mathcal{S}_{r_{0}}$ and that are odd about the equator, are invariant under the time evolution of the system, it follows that the fixed-point subspaces for each of the bifurcating steady solutions that are odd about the equator are also invariant under the time evolution of the system restricted to $\mathcal{U}$. Hence these fixed-point subspaces can be considered as invariant under the time evolution of the system on the surface of the hemisphere $\mathcal{H}_{r_{0}}$. Thus using only the symmetry properties of the model, it is predicted that generically there are precisely three types of bifurcating solutions for the hyperchirality model on the hemisphere, corresponding to the patterns represented by Figures 2, 8 and 9. Furthermore, each steady solution belongs to a linear subspace that is invariant under the time evolution of the hyperchirality model (9)-(10).

In order to verify that the bifurcation is indeed generic, and also to determine the stability of the bifurcating steady solutions, we reduce the hyper- 


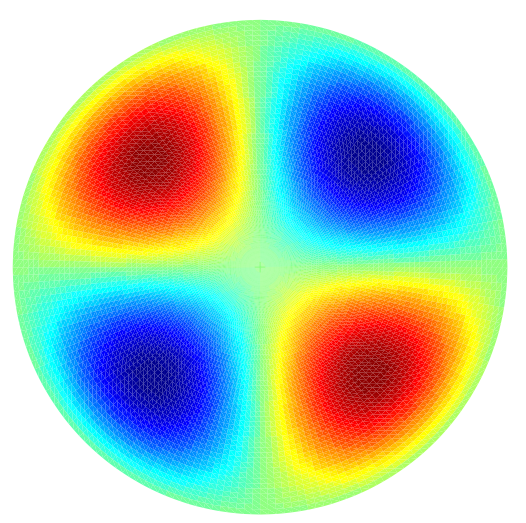

Figure 8: Dichotomous branching pattern for the hyperchirality model, corresponding to the isotropy group $\mathrm{O}^{-}$. This pattern is quite close to $Y_{3,2}$. While branching processes are diverse in their details, Figures 1 and 9 are probably better representations of the commonest growth patterns than is this figure.

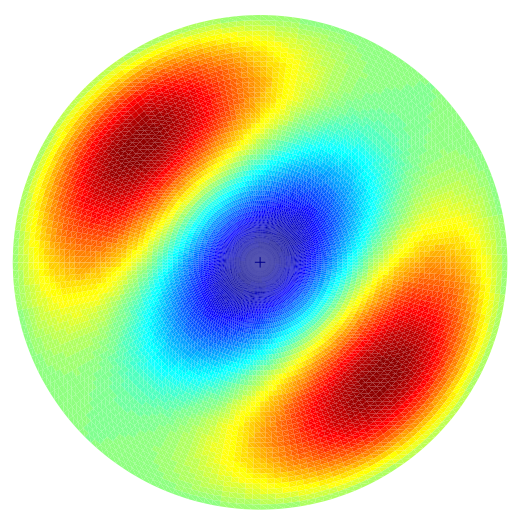

Figure 9: Sector pattern for the hyperchirality model, corresponding to the isotropy group $\mathbf{D}_{6}^{d}$. This pattern is quite close to a particular linear combination of $Y_{3,0}$ and $Y_{3,2}$. 
chirality model to the bifurcation equations (11). Following the procedure outlined in Section 2, we obtain expressions for the coefficients in (11). Due to the absence of quadratic reaction terms (37) in the hyperchirality model, we have

$$
B_{1}=B_{2}=B_{3}=0 \text {, }
$$

thus the reduced equations representing the dynamics on the local centre manifold are

$$
\begin{aligned}
& \dot{x}=x\left(\sigma_{\max }+C_{1} x^{2}+C_{2}|z|^{2}\right), \\
& \dot{z}=z\left(\sigma_{\max }+C_{3} x^{2}+C_{4}|z|^{2}\right), \\
& \dot{\bar{z}}=\bar{z}\left(\sigma_{\max }+C_{3} x^{2}+C_{4}|z|^{2}\right) .
\end{aligned}
$$

From the cubic reaction terms (38) we obtain numerical values for the coefficients in (44),

$$
C_{1}=-0.0911, \quad C_{2}=-0.200, \quad C_{3}=-0.100, \quad C_{4}=-0.170 .
$$

See the Appendix for more details.

The bifurcation analysis is simpler than in the Brusselator case, due to the vanishing of the quadratic coefficients. Symmetry also simplifies computations: since the centre manifold reduction preserves symmetry, it also preserves the fixed-point subspaces of the bifurcating steady solutions.

The fixed-point subspace for the pair of annular patterns is represented in the reduced equations (44) by the $x$-axis, which can be seen to be invariant under time evolution. On the $x$-axis we have

$$
\dot{x}=\sigma_{\max } x+C_{1} x^{3}
$$

from which we see, since $C_{1}<0$, that there is a supercritical bifurcation of a pair of nontrivial equilibrium solutions on the $x$-axis, $x= \pm \sigma_{\max }^{1 / 2} /\left(-C_{1}\right)^{1 / 2}$ for $\sigma_{\max }>0$. In the hyperchirality model, these solutions correspond to the two steady annular patterns predicted by the symmetry arguments. The steady annular patterns for the hyperchirality model are given by $\boldsymbol{U}=$ $x \boldsymbol{U}_{3,0}+\mathcal{O}\left(|x|^{2}\right)$, thus to leading order both components of the annular pattern solutions are scalar multiples of the surface harmonic $Y_{3,0}(\theta, \phi)$.

For the dichotomous branching patterns, the fixed-point subspaces are represented in (44) by lines through the origin in the complex $z$-plane, which 
can be seen to be invariant under the time evolution of $(44)(\arg z(t)$ remains constant). On any such line we have

$$
\dot{z}=\sigma_{\max } z+C_{4}|z|^{2} z
$$

which, since $C_{4}<0$, implies that there is a supercritical bifurcation of a circle of equilibrium solutions in the complex $z$-plane with radius $|z|=$ $\sigma_{\max }^{1 / 2} /\left(-C_{4}\right)^{1 / 2}$ when $\sigma_{\max }>0$. Each of these equilibrium solutions corresponds to a steady dichotomous branching pattern in the hyperchirality model, and moving around the circle of equilibria in the $z$-plane corresponds to rotating the patterned solution of the hyperchirality model about the polar axis of the hemisphere. The steady dichotomous branching patterns for the hyperchirality model have the form $\boldsymbol{U}=z \boldsymbol{U}_{3,2}+\bar{z} \boldsymbol{U}_{3,-2}+\mathcal{O}\left(|(z, \bar{z})|^{2}\right)$, thus to leading order both components are linear combinations of the real and imaginary parts of $Y_{3,2}(\theta, \phi)$, or, equivalently, rotations about the polar axis of the real part of $Y_{3,2}(\theta, \phi)$.

The fixed-point subspaces for the sector patterns in the hyperchirality model appear in the reduced equations (44) as straight lines through the origin making an angle $\alpha$ with the $x$-axis, where $\tan \alpha=\left(C_{4}-C_{2}\right)^{1 / 2} /\left(C_{1}-\right.$ $\left.C_{3}\right)^{1 / 2}=(3 / 10)^{1 / 2}$. The dynamics on the line, projected onto the $x$-axis, are given by

$$
\dot{x}=\sigma_{\max } x+\left(C_{1}+\frac{3}{10} C_{2}\right) x^{3},
$$

thus there is a supercritical bifurcation of a pair of circles of equilibrium solutions with $x= \pm \sigma_{\max }^{1 / 2} /\left(-C_{1}-3 C_{2} / 10\right)^{1 / 2}$ and $|z|=(3 / 10)^{1 / 2} \sigma_{\max }^{1 / 2} /\left(-C_{1}-\right.$ $\left.3 C_{2} / 10\right)^{1 / 2}$. Each of these solutions corresponds to a steady sector pattern in the hyperchirality model. Moving around each of the two circles of equilibria corresponds to rotating a sector patterned solution (Figure 9) of the hyperchirality model about the polar axis of the hemisphere, and the solutions on one circle of equilibria are the negatives of the solutions on the other circle. The steady sector patterns are given by $\boldsymbol{U}= \pm(10 / 3)^{1 / 2}|z| \boldsymbol{U}_{3,0}+z \boldsymbol{U}_{3,2}+$ $\bar{z} \boldsymbol{U}_{3,-2}+\mathcal{O}\left(|(z, \bar{z})|^{2}\right)$, thus to leading order the components are scalar multiples of a rotation of $Y_{3,3}(\theta, \phi)$ by $\pi / 2$ radians about an axis through the centre of the sphere and lying in the equatorial plane.

The stability of the bifurcating solutions is determined by linearizing the reduced equations (44) about each of the solutions. We find that the annular and dichotomous branching patterns are stable, and the sector patterns are unstable. See the bifurcation diagram, Figure 10, and the phase portrait 


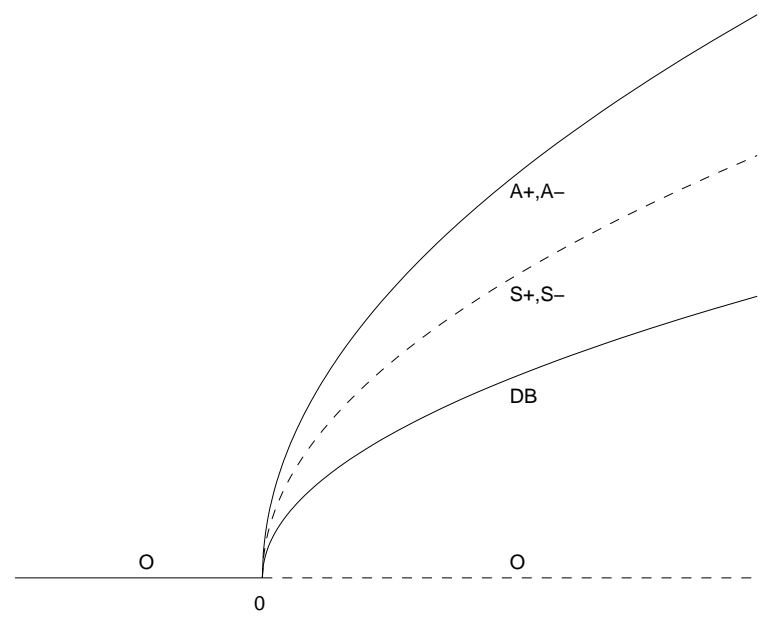

Figure 10: Schematic bifurcation diagram for the hyperchirality model. Values of $\sigma_{\max }$ are plotted along the horizontal axis, and the amplitudes of the equilibrium solutions are plotted along the vertical axis. Solid lines represent branches of stable solutions, and dashed lines represent branches of unstable solutions. $O$ indicates the patternless solution, and $A+, A$ - indicate an annular pattern solution (Figure 2) together with its negative. $D B$ indicates a circle of dichotomous branching pattern solutions (Figure 8). $S+$, $S-$ indicate a pair of circles of sector pattern solutions (Figure 9).

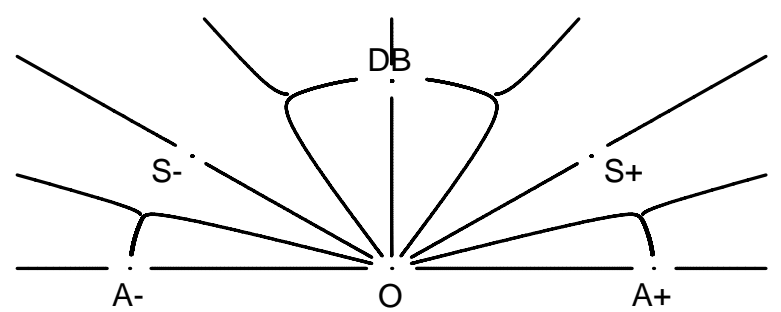

Figure 11: Phase portrait of the bifurcation equations for the hyperchirality model when $\sigma_{\max }>0$. The annular pattern solutions $A+$ and $A-$, and the dichotomous branching pattern solutions $D B$ are stable. The patternless solution $O$ and the sector pattern solutions $S+$ and $S-$ are unstable. 
when $\sigma_{\max }>0$, Figure 11. For $\sigma_{\max }>0$ there are multiple attractors for the hyperchirality model. If we take a random distribution of initial conditions near the patternless steady solution when $\sigma_{\max }>0$, then some will decay to a dichotomous branching pattern, and some will decay to an annular pattern. The basin boundary between the attractors is the double cone $C$ formed in $(x, z)$-space by the fixed-point subspaces of the unstable striped patterns. The vertex of $C$ is the origin and, as noted above, the sides make an angle $\alpha$ with the $x$-axis, where $\tan \alpha=(3 / 10)^{1 / 2}$. The basin of attraction for the pair of annular patterns is the interior of the double cone $C$, which intersects the interior of a sphere of initial conditions centred at the origin occupying a relative volume of $1-\cos \alpha=0.12$ with respect to the volume of the sphere. Thus aproximately $12 \%$ of initial conditions chosen at random near the patternless steady state will decay to one of the two annular patterns when $\sigma_{\max }>0$, and the remaining $88 \%$ will decay to a dichotomous branching pattern. As is the case with the Brusselator model, the coefficients for the hyperchirality model that determine the angle $\alpha$ are all proportional to the same parameter-dependent expression: see equation (73) in the Appendix. Thus $\alpha$ itself does not depend on parameters, and the $88 \%-12 \%$ splitting of a spherically symmetric distribution of initial conditions about the patternless steady state is, to leading order, independent of parameters.

\section{Conclusion}

We have studied two chemical reaction-diffusion systems and shown that either can model the emergence of morphogen prepatterns for dichotomous branching in the growing tips of plants. Both the Brusselator and the hyperchirality reaction-diffusion systems, considered on a hemispherical domain with Dirichlet boundary conditions, undergo bifurcations from a patternless solution to a stable dichotomous branching patterned solution. The two models differ in the details of the dichotomous branching solutions, and in the bifurcation structures. For both models there are also bifurcations of stable annular pattern solutions that represent failure to branch. By estimating the basins of attraction of the two types of solutions for both models we predict the proportions of small random perturbations of the patternless solution that lead to each type of solution: for the Brusselator model $84 \%$ give dichotomous branching and the remaining $16 \%$ give an annular pattern, while for the hyperchirality model $88 \%$ give dichotomous branching and $12 \%$ give 
an annular pattern. For different parameter values, where the patterns are more strongly developed, numerical simulations using the reaction-diffusion equations show roughly similar statistics (Harrison et al., 2001).

In both models, symmetries due to the assumption of a hemispherical domain were used to simplify the analysis. In the hyperchirality model, the presence of only cubic nonlinear reaction terms allowed the problem to be extended to a spherical domain so that additional information on the structure of the patterned solutions was determined by using general results on bifurcations with spherical symmetry.

For the Brusselator model the bifurcation analysis predicts subcritical bifurcation of patterned solutions, so that stable dichotomous branching and annular pattern solutions exist for a range of parameter values for which the patternless solution is also stable. This range of parameter values is rather small (see below) and would likely be missed by simulations if the subcritical bifurcations were not suspected. One consequence of the subcritical bifurcations is that for parameter values just beyond the threshold of instability for the patternless solution, the amplitudes of the stable annular and dichotomous branching patterned solutions are relatively large. For supercritical bifurcations, such as in the hyperchirality model, the amplitudes of the stable patterned solutions are relatively small near the threshold of instability. Numerical simulations of the Brusselator reaction-diffusion system for parameters near the Turing instability boundary show the same qualitative behavior as that predicted by the bifurcation analysis, although the bifurcations occur at somewhat different parameter values than those predicted by our analysis. For parameter values (25)-(26), $r=r_{0}=0.0375$, and $B=1$, the value of $\sigma_{\max }=\sigma_{1}$ for the saddle-node bifurcation of dichotomous branching pattern solutions corresponds to a value of $b=0.363$. Simulations of the Brusselator system locate the saddle-node bifurcation at approximately $b=0.362$. The value of $\sigma_{\max }=\sigma_{2}$ for the saddle-node bifurcation of annular pattern solutions corresponds to $b=0.364$, while simulations locate the same bifurcation at about $b=0.366$. The simulations agree with the location of the transcritical bifurcation predicted at $b=0.367\left(\sigma_{\max }=0\right)$. For the hyperchirality model, simulations of the reaction-diffusion system near the boundary of the Turing instability show close agreement with predictions of the bifurcation analysis.

In the bifurcation analysis, we neglected higher-order terms in the expansions about the bifurcation points. For the Brusselator model, the neglected terms in $(11)$ are $\mathcal{O}\left(\left|\sigma_{\max }\right||(x, z, \bar{z})|^{2}+|(x, z, \bar{z})|^{4}\right)$, while for the hyperchirality 
model, due to the additional symmetry, the neglected higher-order terms are $\mathcal{O}\left(\left|\sigma_{\max }\right||(x, z, \bar{z})|^{3}+|(x, z, \bar{z})|^{5}\right)$. The lower order of terms neglected in the analysis of the Brusselator model is the likely reason for the larger quantitative differences, between the simulations of the reaction-diffusion systems for parameter values near the Turing instability boundary and the predictions of the bifurcation analysis, compared to the hyperchirality model.

Our bifurcation analysis of the Brusselator model could be made more mathematically rigourous if it was done in terms of an unfolding of a degenerate point where the coefficients $B_{1}, B_{2}$ and $B_{3}$ of the quadratic-order terms in the bifurcation equations were all equal to zero. For the parameter values we consider, the numerical values of these coefficients are small, but there is no degenerate point for any parameter values reasonably close to those considered by Harrison et al. (2001). Thus, although our analytical predictions agree qualitatively with numerical simulations for parameter values near the Turing instability as well as farther away, it would be difficult to prove rigourously that this must be the case. However, the calculations leading to the results about percentages of initial conditions do not depend on using degeneracies and unfoldings. The $84 \%-16 \%$ splitting of a spherically symmetric distribution of trajectories starting near the patternless steady state depends only on the lowest-order terms, and it could be proved in a straightforward way that higher-order terms do not affect this splitting. What we have not proved rigourously is that the ultimate destination of these two classes of trajectories, for the Brusselator model, must be a dichotomous branching pattern or an annular pattern.

In estimating the probabilities that a randomly chosen small perturbation of the patternless state will evolve into a dichotomous branching pattern or an annular pattern, we assumed that the decay of modal components of the perturbations in directions transverse to the centre manifold takes place on a much shorter time scale than evolution on the centre manifold itself. Numerical simulations of the full reaction diffusion systems near bifurcation show just such rapid decay, and only minor effects due to components transverse to the centre manifolds. Therefore we have neglected these effects entirely in estimating the probabilities.

In each model the choice of bifurcation parameter is motivated by mathematical convenience - the ease with which the dependence of the largest eigenvalue $\sigma_{\max }$ on the bifurcation parameter could be isolated, in order to find the critical value that makes $\sigma_{\max }=0$. However, a different parameter could be used as a bifurcation parameter, such as the radius $r$ of the hemi- 
spherical domain (i.e. the size of the growing tip), or the concentration $A$ or $B$ of an initial reactant. The only requirement is for $\sigma_{\max }$ to move through zero in a generic way as the bifurcation parameter changes.

In both models, a significant minority of small perturbations of the patternless state evolve, as $t \rightarrow \infty$, to an annular pattern which corresponds to failure to branch. Harrison at al. (2001) discuss the possibility that the earlier parts of transients, rather than later parts, could be more important for morphology, in which case dichotomous branching would be selected more robustly using both models. However, in conventional reaction-diffusion modelling it is assumed that the behavior as $t \rightarrow \infty$ determines the morphological patterns that appear. It is interesting to note that in an experimental study of many genetically identical somatic embryos of a hybrid larch, von Aderkas (2000) reports a distribution in the number (from 0 to 15) of cotelydons forming in the embryos, with about $20 \%$ of them ending in an annular pattern with no cotelydons.

In more realistic models one could have symmetries "broken externally" by removing the idealizations that correspond to the symmetries. For example, the growing tip could be modelled as not exactly hemispherical, and we could consider a more irregular, perhaps more realistic, domain. In the hyperchirality model, the kinematics of the pair of activator substances with concentrations $X_{D}$ and $X_{L}$, or of the pair of inhibitor substances with concentrations $Y_{D}$ and $Y_{L}$ could be modelled as not exactly identical. If we consider only small departures from the idealized conditions that we have assumed in this paper, our predictions are easily modified by considering generic perturbations of the symmetric systems. Isolated stable equilibria such as those corresponding to the annular pattern solutions will persist with only small distortions in the circular symmetry, while the circles of equilibria corresponding to the continuous families of dichotomous branching patterns would typically be perturbed into a pair of such equilibria, one stable and one unstable, that corresponded to dichotomous branching patterns with specific orientations with respect to the domain. The circles of equilibria would be replaced by invariant circles containing equilibria, with very slow evolution along the circles between the equilibria. The corresponding solutions of the reaction-diffusion models would evolve relatively quickly into something resembling a dichotomous branching pattern, then there would be a very slow rotation of the pattern towards a specific orientation. The proportions of small random perturbations of the patternless state that eventually approach an annular pattern or a dichotomous branching pattern would re- 
main approximately the same. In general, compared to a direct study of a model that lacks symmetry, analysis of a nearly symmetric model is easier and more information is gained if we assume symmetry is present, exploit the symmetry to obtain results, then modify the results to take account of generic perturbations that break the symmetry.

We have considered behavior in the two models for parameters close to the threshold of instability of the patternless solution, i.e. the Turing instability boundary. The bifurcations of solutions represent the initial appearance of reaction-diffusion patterns in a growing tip of a plant, and we have shown that both models predict the coexistence of stable final patterned states, both annular and dichotomously branched. Harrison et al. (2001) use numerical simulation to investigate behavior for parameters farther from the Turing instability boundary, for which patterns of various kinds are more strongly developed. For the Brusselator and hyperchirality models, randomly chosen initial conditions give rise to annular patterns in roughly $20 \%$ of cases, a somewhat larger proportion than what we have predicted analytically for parameters close to the Turing instability boundary. In this paper, attention is restricted to a hemispherical tip whose shape is unchanged. Harrison et al. (2001) discuss incorporating the reaction-diffusion patterning into a larger model that includes morphological change, following growing tips in three dimensions that branch, change shape and grow sequentially to form repeatedly branched structures that can model plant growth.

\section{A Appendix: Reduction to bifurcation equa- tions}

In this Appendix, we give more details of the calculations used to find values for the coefficients $B_{1}, B_{2}, B_{3}, C_{1}, C_{2}, C_{3}$ and $C_{4}$ in the bifurcation equations (11) that govern the dynamics on the centre manifold.

We observe that $\boldsymbol{A}\left(\lambda_{0}\right) \boldsymbol{U}^{c}=\mathbf{0}$ for any vector function $\boldsymbol{U}^{c}$ belonging to the centre eigenspace $E^{c}$ given by (14). The span of the eigenfunctions corresponding to the remaining, infinitely many, eigenvalues with negative real parts, is the stable eigenspace $E^{s}$. The stable eigenspace is invariant under $\boldsymbol{A}\left(\lambda_{0}\right)$, i.e., if a vector function $\boldsymbol{U}^{s}$ belongs to $E^{s}$, then so does $\boldsymbol{A}\left(\lambda_{0}\right) \boldsymbol{U}^{s}$.

For two vector functions $\boldsymbol{U}_{j}=\left(U_{j}(\theta, \phi), V_{j}(\theta, \phi)\right)^{T}, j=1,2$, we define 
their inner product by

$$
\left\langle\boldsymbol{U}_{1}, \boldsymbol{U}_{2}\right\rangle=\int_{0}^{2 \pi} \int_{0}^{\pi / 2}\left[U_{1}(\theta, \phi) \overline{U_{2}(\theta, \phi)}+V_{1}(\theta, \phi) \overline{V_{2}(\theta, \phi)}\right] \sin \theta d \theta d \phi
$$

and the corresponding $L^{2}$-norm of a vector function by

$$
\|\boldsymbol{U}\|=\langle\boldsymbol{U}, \boldsymbol{U}\rangle^{1 / 2}=\left\{\int_{0}^{2 \pi} \int_{0}^{\pi / 2}\left[U(\theta, \phi)^{2}+V(\theta, \phi)^{2}\right] \sin \theta d \theta d \phi\right\}^{1 / 2} .
$$

For any $\lambda$, the adjoint operator to $\boldsymbol{A}(\lambda)$ is defined to be the linear operator $\boldsymbol{A}(\lambda)^{*}$ that satisfies

$$
\left\langle\boldsymbol{A}(\lambda) \boldsymbol{U}, \boldsymbol{U}^{*}\right\rangle=\left\langle\boldsymbol{U}, \boldsymbol{A}(\lambda)^{*} \boldsymbol{U}^{*}\right\rangle
$$

for all vector functions $\boldsymbol{U}$ and $\boldsymbol{U}^{*}$. It then follows that the adjoint eigenvalue problem

$$
\boldsymbol{A}(\lambda)^{*} \boldsymbol{U}^{*}=\sigma \boldsymbol{U}^{*}
$$

has the same eigenvalues as the eigenvalue problem (13), and corresponding to the zero eigenvalue for (51) when $\lambda=\lambda_{0}$ are three linearly independent adjoint vector eigenfunctions, which we may choose as

$$
\boldsymbol{U}_{3,0}^{*}, \quad \boldsymbol{U}_{3,2}^{*}, \quad \boldsymbol{U}_{3,-2}^{*},
$$

so that orthonormality conditions

$$
\left\langle\boldsymbol{U}_{3, m_{1}}, \boldsymbol{U}_{3, m_{2}}^{*}\right\rangle=\delta_{m_{1} m_{2}}
$$

are satisfied, where $\delta_{m_{1} m_{2}}$ is the Kronecker delta. Moreover, each of the adjoint eigenfunctions $\boldsymbol{U}_{3, m}^{*}, m=0, \pm 2$, is orthogonal to any vector function in the stable eigenspace $E^{s}$. The linear operator $\boldsymbol{P}^{c}$ defined by

$$
\boldsymbol{P}^{c} \boldsymbol{U}=x \boldsymbol{U}_{3,0}+z \boldsymbol{U}_{3,2}+\bar{z} \boldsymbol{U}_{3,-2},
$$

where

$$
x=\left\langle\boldsymbol{U}, \boldsymbol{U}_{3,0}^{*}\right\rangle, \quad z=\left\langle\boldsymbol{U}, \boldsymbol{U}_{3,2}^{*}\right\rangle, \quad \bar{z}=\left\langle\boldsymbol{U}, \boldsymbol{U}_{3,-2}^{*}\right\rangle
$$

for any vector function $\boldsymbol{U}$, is a projection onto the centre eigenspace $E^{c}$, with null space equal to the stable eigenspace $E^{s}$. Then $\boldsymbol{I}-\boldsymbol{P}^{c}$, where $\boldsymbol{I}$ is the identity operator, is a projection onto $E^{s}$, with null space equal to $E^{c}$. Both the projections $\boldsymbol{P}^{c}$ and $\boldsymbol{I}-\boldsymbol{P}^{c}$ commute with $\boldsymbol{A}\left(\lambda_{0}\right)$. 
We use centre manifold theory to express a vector function lying in the local centre manifold $W_{l o c}^{c}$, when $\lambda=\lambda_{0}$, as a Taylor series

$$
\begin{aligned}
& \boldsymbol{U}=x \boldsymbol{U}_{3,0}+z \boldsymbol{U}_{3,2}+\bar{z} \boldsymbol{U}_{3,-2}+x^{2} \boldsymbol{U}_{(200)}+x z \boldsymbol{U}_{(110)}+x \bar{z} \boldsymbol{U}_{(101)} \\
& +z^{2} \boldsymbol{U}_{(020)}+z \bar{z} \boldsymbol{U}_{(011)}+\bar{z}^{2} \boldsymbol{U}_{(002)}+\mathcal{O}\left(|(x, z, \bar{z})|^{3}\right)
\end{aligned}
$$

in the small parameters $(x, z, \bar{z})$. The vector functions $\boldsymbol{U}_{\left(j_{1} j_{2} j_{3}\right)}$ with $j_{1}+$ $j_{2}+j_{3} \geq 2$ all belong to the stable eigenspace $E^{s}$ and can be calculated by substituting the expansion (53) into the projection $\boldsymbol{I}-\boldsymbol{P}^{c}$ of the system (12) onto $E^{s}$ when $\lambda=\lambda_{0}$, and collecting terms in powers of $x, z$ and $\bar{z}$. For example, collecting terms in $x^{2}$ gives

$$
\mathbf{0}=\boldsymbol{A}\left(\lambda_{0}\right) \boldsymbol{U}_{(200)}+\left(\boldsymbol{I}-\boldsymbol{P}^{c}\right) \boldsymbol{B}\left(\lambda_{0} ; \boldsymbol{U}_{3,0}, \boldsymbol{U}_{3,0}\right) .
$$

This is a nonhomogeneous system of linear partial differential equations, which may be solved for $\boldsymbol{U}_{(200)}$. The other vector functions $\boldsymbol{U}_{(110)}, \boldsymbol{U}_{(101)}$, etc. in (53) can be computed similarly. It turns out that only a few of the vector functions $\boldsymbol{U}_{\left(j_{1} j_{2} j_{3}\right)}$ are needed explicitly for the Brusselator model, and none of them are needed for the hyperchirality model.

The three ordinary differential equations that govern the dynamics on the local centre manifold $W_{l o c}^{c}$ are found by substituting the expansion (53) into the full system (12) and then taking inner products with each of the three adjoint eigenfunctions $\boldsymbol{U}_{3,0}^{*}, \boldsymbol{U}_{3,2}^{*}$ and $\boldsymbol{U}_{3,-2}^{*}$. To determine bifurcation and stability of solutions when $\lambda$ is near $\lambda_{0}$, it is sufficient to expand (12) about $\lambda_{0}$, neglecting higher-order dependence of the nonlinear parts $\boldsymbol{B}$ and $\boldsymbol{C}$ on $\lambda$. Thus we obtain the system of ordinary differential equations

$$
\begin{aligned}
& \dot{x}=\left\langle\boldsymbol{A}\left(\lambda_{0}\right) \boldsymbol{U}+\left(\lambda-\lambda_{0}\right) \boldsymbol{A}^{\prime}\left(\lambda_{0}\right) \boldsymbol{U}+\boldsymbol{B}\left(\lambda_{0} ; \boldsymbol{U}, \boldsymbol{U}\right)+\boldsymbol{C}\left(\lambda_{0} ; \boldsymbol{U}, \boldsymbol{U}, \boldsymbol{U}\right), \boldsymbol{U}_{3,0}^{*}\right\rangle, \\
& \dot{z}=\left\langle\boldsymbol{A}\left(\lambda_{0}\right) \boldsymbol{U}+\left(\lambda-\lambda_{0}\right) \boldsymbol{A}^{\prime}\left(\lambda_{0}\right) \boldsymbol{U}+\boldsymbol{B}\left(\lambda_{0} ; \boldsymbol{U}, \boldsymbol{U}\right)+\boldsymbol{C}\left(\lambda_{0} ; \boldsymbol{U}, \boldsymbol{U}, \boldsymbol{U}\right), \boldsymbol{U}_{3,2}^{*}, 4\right) \\
& \dot{\bar{z}}=\left\langle\boldsymbol{A}\left(\lambda_{0}\right) \boldsymbol{U}+\left(\lambda-\lambda_{0}\right) \boldsymbol{A}^{\prime}\left(\lambda_{0}\right) \boldsymbol{U}+\boldsymbol{B}\left(\lambda_{0} ; \boldsymbol{U}, \boldsymbol{U}\right)+\boldsymbol{C}\left(\lambda_{0} ; \boldsymbol{U}, \boldsymbol{U}, \boldsymbol{U}\right), \boldsymbol{U}_{3,-2}^{*}\right\rangle,
\end{aligned}
$$

where $\boldsymbol{U}$ is given by the expansion (53).

The linear terms in (54) simplify if we recall that $\boldsymbol{A}\left(\lambda_{0}\right)$ applied to any of the eigenfunctions in $E^{c}$ gives zero, and that $\boldsymbol{A}\left(\lambda_{0}\right)$ applied to any vector function in $E^{s}$ remains in $E^{s}$, hence is orthogonal to any adjoint eigenfunction $\boldsymbol{U}_{3, m}^{*}$. Further simplification is obtained by approximating $\left(\lambda-\lambda_{0}\right) \boldsymbol{A}^{\prime}\left(\lambda_{0}\right) \boldsymbol{U}_{3, m}$ by $\sigma_{m a x} \boldsymbol{U}_{3, m}$ when $\boldsymbol{U}_{3, m}$ is an eigenfunction in $E^{c}$, where $\sigma_{\max }=\mathcal{O}\left(\left|\lambda-\lambda_{0}\right|\right)$ is the largest eigenvalue of the eigenvalue problem (13) when $\lambda$ is near $\lambda_{0}\left(\sigma_{\max }\right.$ is the growth rate of the fastest growing modes). 
When $\lambda$ is near $\lambda_{0}$, we have $\left.i\right) \sigma_{\max }<0$ if $\left.\lambda<\lambda_{0}, i i\right) \sigma_{\max }=0$ if $\lambda=\lambda_{0}$, and iii) $\sigma_{\max }>0$ if $\lambda>\lambda_{0}$. With these considerations, the system of ordinary differential equations (54) becomes

$$
\begin{aligned}
& \dot{x}=\sigma_{\max } x+\left\langle\boldsymbol{B}\left(\lambda_{0} ; \boldsymbol{U}, \boldsymbol{U}\right), \boldsymbol{U}_{3,0}^{*}\right\rangle+\left\langle\boldsymbol{C}\left(\lambda_{0} ; \boldsymbol{U}, \boldsymbol{U}, \boldsymbol{U}\right), \boldsymbol{U}_{3,0}^{*}\right\rangle, \\
& \dot{z}=\sigma_{\max } z+\left\langle\boldsymbol{B}\left(\lambda_{0} ; \boldsymbol{U}, \boldsymbol{U}\right), \boldsymbol{U}_{3,2}^{*}\right\rangle+\left\langle\boldsymbol{C}\left(\lambda_{0} ; \boldsymbol{U}, \boldsymbol{U}, \boldsymbol{U}\right), \boldsymbol{U}_{3,2}^{*}\right\rangle, \\
& \dot{\bar{z}}=\sigma_{\max } \bar{z}+\left\langle\boldsymbol{B}\left(\lambda_{0} ; \boldsymbol{U}, \boldsymbol{U}\right), \boldsymbol{U}_{3,-2}^{*}\right\rangle+\left\langle\boldsymbol{C}\left(\lambda_{0} ; \boldsymbol{U}, \boldsymbol{U}, \boldsymbol{U}\right), \boldsymbol{U}_{3 .-2}^{*}\right\rangle,
\end{aligned}
$$

where we neglect higher-order terms $\mathcal{O}\left(\left|\lambda-\lambda_{0}\right|^{2}|(x, z, \bar{z})|+\left|\lambda-\lambda_{0}\right||(x, z, \bar{z})|^{2}\right)$. Substituting the Taylor series (53) for $\boldsymbol{U}$ into (55), we expand and then collect terms in powers of $x, z$ and $\bar{z}$. For example, collecting terms in $x^{2}$ in the equation for $\dot{x}$, we obtain

$$
\dot{x}=\sigma_{\max } x+B_{1} x^{2}+\cdots,
$$

where the coefficient of $x^{2}$ is given by the integral

$$
B_{1}=\left\langle\boldsymbol{B}\left(\lambda_{0} ; \boldsymbol{U}_{3,0}, \boldsymbol{U}_{3,0}\right), \boldsymbol{U}_{3,0}^{*}\right\rangle .
$$

For the Brusselator, the eigenfunctions of the eigenvalue problem (22) that correspond to the eigenvalue $\sigma_{\max }=0$ at the critical parameter values are

$$
\begin{gathered}
\boldsymbol{U}_{3,0}=N_{3,0}\left(\begin{array}{c}
k_{2} Y_{3,0}(\theta, \phi) \\
k_{5} Y_{3,0}(\theta, \phi)
\end{array}\right), \\
\boldsymbol{U}_{3,2}=N_{3,2}\left(\begin{array}{c}
k_{2} Y_{3,2}(\theta, \phi) \\
k_{5} Y_{3,2}(\theta, \phi)
\end{array}\right), \\
\boldsymbol{U}_{3,-2}=N_{3,-2}\left(\begin{array}{c}
k_{2} Y_{3,-2}(\theta, \phi) \\
k_{5} Y_{3,-2}(\theta, \phi)
\end{array}\right),
\end{gathered}
$$

where

$$
k_{5}=\omega^{2} D_{X}-k_{1},
$$

and the normalization constants $N_{3, m}$ are chosen as

$$
N_{3,0}=\sqrt{\frac{7}{2 \pi\left(k_{2}^{2}+k_{5}^{2}\right)}}, \quad N_{3,2}=N_{3,-2}=\sqrt{\frac{7}{240 \pi\left(k_{2}^{2}+k_{5}^{2}\right)}},
$$

so that the $L^{2}$-norms of the eigenfunctions satisfy

$$
\left\|\boldsymbol{U}_{3, m}\right\|=1, \quad m=0, \pm 2 .
$$


The corresponding adjoint eigenfunctions are

$$
\begin{gathered}
\boldsymbol{U}_{3,0}^{*}=N_{3,0}^{*}\left(\begin{array}{c}
k_{3} Y_{3,0}(\theta, \phi) \\
k_{5} Y_{3,0}(\theta, \phi)
\end{array}\right), \\
\boldsymbol{U}_{3,2}^{*}=N_{3,2}^{*}\left(\begin{array}{c}
k_{3} Y_{3,2}(\theta, \phi) \\
k_{5} Y_{3,2}(\theta, \phi)
\end{array}\right), \\
\boldsymbol{U}_{3,-2}^{*}=N_{3,-2}^{*}\left(\begin{array}{c}
k_{3} Y_{3,-2}(\theta, \phi) \\
k_{5} Y_{3,-2}(\theta, \phi)
\end{array}\right),
\end{gathered}
$$

where the normalization constants $N_{3, m}^{*}$ are chosen as

$$
N_{3,0}^{*}=\frac{\sqrt{7\left(k_{2}^{2}+k_{5}^{2}\right)}}{\sqrt{2 \pi}\left(k_{2} k_{3}+k_{5}^{2}\right)}, \quad N_{3,2}^{*}=N_{3,-2}^{*}=\frac{\sqrt{7\left(k_{2}^{2}+k_{5}^{2}\right)}}{\sqrt{240 \pi}\left(k_{2} k_{3}+k_{5}^{2}\right)}
$$

so that the inner products of the adjoint eigenfunctions with the eigenfunctions satisfy the orthonormalization conditions (52).

In order to find the coefficients $B_{1}, B_{2}, B_{3}$ for the quadratic terms in (11) for the Brusselator model, it suffices to take

$$
\boldsymbol{U}=x \boldsymbol{U}_{3,0}+z \boldsymbol{U}_{3,2}+\bar{z} \boldsymbol{U}_{3,-2}
$$

in (55), since only quadratic terms are needed. Thus

$$
\begin{aligned}
& B_{1}=\left\langle\boldsymbol{B}\left(\lambda_{0} ; \boldsymbol{U}_{3,0}, \boldsymbol{U}_{3,0}\right), \boldsymbol{U}_{3,0}^{*}\right\rangle, \\
& B_{2}=\left\langle\boldsymbol{B}\left(\lambda_{0} ; \boldsymbol{U}_{3,2}, \boldsymbol{U}_{3,-2}\right)+\boldsymbol{B}\left(\lambda_{0} ; \boldsymbol{U}_{3,-2}, \boldsymbol{U}_{3,2}\right), \boldsymbol{U}_{3,0}^{*}\right\rangle, \\
& B_{3}=\left\langle\boldsymbol{B}\left(\lambda_{0} ; \boldsymbol{U}_{3,0}, \boldsymbol{U}_{3,2}\right)+\boldsymbol{B}\left(\lambda_{0} ; \boldsymbol{U}_{3,2}, \boldsymbol{U}_{3,0}\right), \boldsymbol{U}_{3,2}^{*}\right\rangle .
\end{aligned}
$$

Using expressions (20), (56) and (58) for $\boldsymbol{B}, \boldsymbol{U}_{3, m}$ and $\boldsymbol{U}_{3, m}^{*}$ in (60), we evaluate the inner products using (49), and obtain

$$
B_{1}=\frac{7 \sqrt{14}}{128 \sqrt{\pi}} \mu, \quad B_{2}=-\frac{35 \sqrt{14}}{128 \sqrt{\pi}} \mu, \quad B_{3}=-\frac{35 \sqrt{14}}{128 \sqrt{\pi}} \mu,
$$

where

$$
\mu=\left(\frac{d b B}{a A} k_{2}+\frac{2 c a A}{d} k_{7}\right) \frac{k_{2}\left(k_{3}-k_{7}\right)}{\left(k_{2} k_{3}+k_{7}^{2}\right) \sqrt{k_{2}^{2}+k_{7}^{2}}} .
$$

For parameter values (25)-(27) we obtain the numerical values (30) given in Section 3.2. 
To find the coefficients $C_{1}, C_{2}, C_{3}, C_{4}$ of the cubic terms in (11) for the Brusselator requires more work. Substituting the expansion (53) for $\boldsymbol{U}$ into (55), and collecting cubic terms in $(x, z, \bar{z})$, we obtain

$$
\begin{aligned}
C_{1}=\langle & \left.\boldsymbol{C}\left(\lambda_{0} ; \boldsymbol{U}_{3,0}, \boldsymbol{U}_{3,0}, \boldsymbol{U}_{3,0}\right)+\boldsymbol{B}\left(\lambda_{0} ; \boldsymbol{U}_{3,0}, \boldsymbol{U}_{(200)}\right), \boldsymbol{U}_{3,0}^{*}\right\rangle \\
C_{2}=\langle & \boldsymbol{C}\left(\lambda_{0} ; \boldsymbol{U}_{3,0}, \boldsymbol{U}_{3,2}, \boldsymbol{U}_{3,-2}\right)+\boldsymbol{B}\left(\lambda_{0} ; \boldsymbol{U}_{3,0}, \boldsymbol{U}_{(011)}\right)+\boldsymbol{B}\left(\lambda_{0} ; \boldsymbol{U}_{3,2}, \boldsymbol{U}_{(101)}\right) \\
& \left.+\boldsymbol{B}\left(\lambda_{0} ; \boldsymbol{U}_{3,-2}, \boldsymbol{U}_{(110)}\right), \boldsymbol{U}_{3,0}^{*}\right\rangle \\
C_{3}=\langle & \boldsymbol{C}\left(\lambda_{0} ; \boldsymbol{U}_{3,0}, \boldsymbol{U}_{3,0}, \boldsymbol{U}_{3,2}\right)+\boldsymbol{B}\left(\lambda_{0} ; \boldsymbol{U}_{3,0}, \boldsymbol{U}_{(110)}\right) \\
& \left.+\boldsymbol{B}\left(\lambda_{0} ; \boldsymbol{U}_{3,2}, \boldsymbol{U}_{(200)}\right), \boldsymbol{U}_{3,2}^{*}\right\rangle \\
C_{4}=\langle & \boldsymbol{C}\left(\lambda_{0} ; \boldsymbol{U}_{3,2}, \boldsymbol{U}_{3,2}, \boldsymbol{U}_{3,-2}\right)+\boldsymbol{B}\left(\lambda_{0} ; \boldsymbol{U}_{3,2}, \boldsymbol{U}_{(011)}\right) \\
& \left.+\boldsymbol{B}\left(\lambda_{0} ; \boldsymbol{U}_{3,-2}, \boldsymbol{U}_{(020)}\right), \boldsymbol{U}_{3,2}^{*}\right\rangle .
\end{aligned}
$$

We do not need all of the $\boldsymbol{U}_{\left(j_{1} j_{2} j_{3}\right)}$ explicitly, but only $\boldsymbol{U}_{(200)}, \boldsymbol{U}_{(110)}, \boldsymbol{U}_{(101)}$, $\boldsymbol{U}_{(011)}$ and $\boldsymbol{U}_{(020)}$.

The vector functions $\boldsymbol{U}_{\left(j_{1} j_{2} j_{3}\right)}$ can be found by substituting the Taylor series (53) into the projection $\boldsymbol{I}-\boldsymbol{P}^{c}$ of the system (12) onto the stable eigenspace $E^{s}$ when $\lambda=\lambda_{0}$, and then collecting terms in powers of $(x, z, \bar{z})$. For example, collecting terms in $x^{2}$ gives

$$
\boldsymbol{A}\left(\lambda_{0}\right) \boldsymbol{U}_{(200)}=-\left(\boldsymbol{I}-\boldsymbol{P}^{c}\right) \boldsymbol{B}\left(\lambda_{0} ; \boldsymbol{U}_{3,0}, \boldsymbol{U}_{3,0}\right),
$$

while collecting terms in $x z$ gives

$$
\boldsymbol{A}\left(\lambda_{0}\right) \boldsymbol{U}_{(110)}=-\left(\boldsymbol{I}-\boldsymbol{P}^{c}\right) \boldsymbol{B}\left(\lambda_{0} ; \boldsymbol{U}_{3,0}, \boldsymbol{U}_{3,2}\right) .
$$

Similarly, we get

$$
\begin{gathered}
\boldsymbol{A}\left(\lambda_{0}\right) \boldsymbol{U}_{(101)}=-\left(\boldsymbol{I}-\boldsymbol{P}^{c}\right) \boldsymbol{B}\left(\lambda_{0} ; \boldsymbol{U}_{3,0}, \boldsymbol{U}_{3,-2}\right), \\
\boldsymbol{A}\left(\lambda_{0}\right) \boldsymbol{U}_{(011)}=-\left(\boldsymbol{I}-\boldsymbol{P}^{c}\right)\left[\boldsymbol{B}\left(\lambda_{0} ; \boldsymbol{U}_{3,2}, \boldsymbol{U}_{3,-2}\right),\right.
\end{gathered}
$$

and

$$
\boldsymbol{A}\left(\lambda_{0}\right) \boldsymbol{U}_{(020)}=-\left(\boldsymbol{I}-\boldsymbol{P}^{c}\right) \boldsymbol{B}\left(\lambda_{0} ; \boldsymbol{U}_{3,2}, \boldsymbol{U}_{3,2}\right) .
$$

Equations (63)-(67) are nonhomogeneous systems of linear partial differential equations, which may be solved for the $\boldsymbol{U}_{\left(j_{1} j_{2} j_{3}\right)}$.

To solve the equations, we substitute the explicit expressions (56) for the Brusselator eigenfunctions into the right hand sides of (63)-(67), and, using the expression (20) for $\boldsymbol{B}$, expand in series of surface harmonics which 
satisfy the Dirichlet boundary condition. The projection $\boldsymbol{I}-\boldsymbol{P}^{c}$ affects only those nonzero terms in the expansions corresponding to $l=3$ and $m=0$ or \pm 2 . By expanding both components of the required $\boldsymbol{U}_{\left(j_{1} j_{2} j_{3}\right)}$ in series of the same surface harmonics, the coefficients are solved for term by term. The resulting series expressions for the required $\boldsymbol{U}_{\left(j_{1} j_{2} j_{3}\right)}$ are used, together with the expressions (20), (21), (56) and (58) for $\boldsymbol{B}, \boldsymbol{C}, \boldsymbol{U}_{3, m}$ and $\boldsymbol{U}_{3, m}^{*}$, in the inner products (62) to obtain series of real numbers for the cubic coeffients $C_{1}, C_{2}, C_{3}, C_{4}$. We use the computer algebra program Maple to evaluate the terms in each series and then to sum the terms numerically. We accept the series as converged when additional terms in the series do not affect the sum to ten significant digits, and obtain the values (31) given in Section 3.2.

For the hyperchirality model, the eigenfunctions for the eigenvalue $\sigma_{\max }=$ 0 at the critical parameter values are

$$
\begin{gathered}
\boldsymbol{U}_{3,0}=N_{3,0}\left(\begin{array}{c}
k_{2} Y_{3,0}(\theta, \phi) \\
k_{7} Y_{3,0}(\theta, \phi)
\end{array}\right), \\
\boldsymbol{U}_{3,2}=N_{3,2}\left(\begin{array}{c}
k_{2} Y_{3,2}(\theta, \phi) \\
k_{7} Y_{3,2}(\theta, \phi)
\end{array}\right), \\
\boldsymbol{U}_{3,-2}=N_{3,-2}\left(\begin{array}{c}
k_{2} Y_{3,-2}(\theta, \phi) \\
k_{7} Y_{3,-2}(\theta, \phi)
\end{array}\right),
\end{gathered}
$$

where

$$
k_{7}=\omega^{2} D_{X}-k_{1 a}-k_{1 b},
$$

and the normalization constants $N_{3, m}$ are chosen as

$$
N_{3,0}=\sqrt{\frac{7}{2 \pi\left(k_{2}^{2}+k_{7}^{2}\right)}}, \quad N_{3,2}=N_{3,-2}=\sqrt{\frac{7}{240 \pi\left(k_{2}^{2}+k_{7}^{2}\right)}},
$$

so that

$$
\left\|\boldsymbol{U}_{3, m}\right\|=1, \quad m=0, \pm 2 .
$$

The adjoint eigenfunctions are chosen as

$$
\begin{gathered}
\boldsymbol{U}_{3,0}^{*}=N_{3,0}^{*}\left(\begin{array}{c}
k_{3} Y_{3,0}(\theta, \phi) \\
k_{7} Y_{3,0}(\theta, \phi)
\end{array}\right), \\
\boldsymbol{U}_{3,2}^{*}=N_{3,2}^{*}\left(\begin{array}{c}
k_{3} Y_{3,2}(\theta, \phi) \\
k_{7} Y_{3,2}(\theta, \phi)
\end{array}\right), \\
\boldsymbol{U}_{3,-2}^{*}=N_{3,-2}^{*}\left(\begin{array}{c}
k_{3} Y_{3,-2}(\theta, \phi) \\
k_{7} Y_{3,-2}(\theta, \phi)
\end{array}\right),
\end{gathered}
$$


where

$$
N_{3,0}^{*}=\frac{\sqrt{7\left(k_{2}^{2}+k_{7}^{2}\right)}}{\sqrt{2 \pi}\left(k_{2} k_{3}+k_{7}^{2}\right)}, \quad N_{3,2}^{*}=N_{3,-2}^{*}=\frac{\sqrt{7\left(k_{2}^{2}+k_{7}^{2}\right)}}{\sqrt{240 \pi}\left(k_{2} k_{3}+k_{7}^{2}\right)},
$$

so that the orthonormalization conditions (52) are satisfied.

The computations to determine the coefficients of the equations (11) governing the dynamics on the centre manifold for the hyperchirality model are much shorter, since there are no quadratic reaction terms in the reactiondiffusion system (9), thus $\boldsymbol{B}=\mathbf{0}$ in (12). We immediately have

$$
B_{1}=B_{2}=B_{3}=0,
$$

and the Taylor series (53) is known to be

$$
\boldsymbol{U}=x \boldsymbol{U}_{3,0}+z \boldsymbol{U}_{3,2}+\bar{z} \boldsymbol{U}_{3,-2}+\mathcal{O}\left(|(x, z, \bar{z})|^{3}\right) .
$$

The expressions for the cubic coefficients in (11) simplify from (62) to

$$
\begin{aligned}
& C_{1}=\left\langle\boldsymbol{C}\left(\lambda_{0} ; \boldsymbol{U}_{3,0}, \boldsymbol{U}_{3,0}, \boldsymbol{U}_{3,0}\right), \boldsymbol{U}_{3,0}^{*}\right\rangle, \\
& C_{2}=\left\langle\boldsymbol{C}\left(\lambda_{0} ; \boldsymbol{U}_{3,0}, \boldsymbol{U}_{3,2}, \boldsymbol{U}_{3,-2}\right), \boldsymbol{U}_{3,0}^{*}\right\rangle, \\
& C_{3}=\left\langle\boldsymbol{C}\left(\lambda_{0} ; \boldsymbol{U}_{3,0}, \boldsymbol{U}_{3,0}, \boldsymbol{U}_{3,2}\right), \boldsymbol{U}_{3,2}^{*}\right\rangle, \\
& C_{4}=\left\langle\boldsymbol{C}\left(\lambda_{0} ; \boldsymbol{U}_{3,2}, \boldsymbol{U}_{3,2}, \boldsymbol{U}_{3,-2}\right), \boldsymbol{U}_{3,2}^{*}\right\rangle .
\end{aligned}
$$

By using the expressions (38), (68) and (70) for $\boldsymbol{C}, \boldsymbol{U}_{3, m}$ and $\boldsymbol{U}_{3, m}^{*}$ in (72), the inner products give

$$
C_{1}=-\frac{1687}{1430 \pi} \nu, \quad C_{2}=-\frac{371}{143 \pi} \nu, \quad C_{3}=-\frac{371}{286 \pi} \nu, \quad C_{4}=-\frac{315}{143 \pi} \nu,
$$

where

$$
\nu=\frac{k_{1 a} k_{2}^{3} k_{3}+k_{5} k_{2} k_{7}^{2} k_{3}+k_{6} k_{2}^{2} k_{7}^{2}}{\left(k_{2}^{2}+k_{7}^{2}\right)\left(k_{2} k_{3}+k_{7}^{2}\right)} .
$$

Evaluating the expressions (73) at the critical parameter values give the numerical values (45) stated in Section 3.3.

\section{References}

[1] J. Carr (1981). Applications of Centre Manifold Theory, New York: Springer. 
[2] P. Chossat, R. Lauterbach and I. Melbourne (1990). Steady-state bifurcation with $O(3)$-symmetry. Arch. Rational Mech. Anal. 113, 313-376.

[3] G. Dewel, P. Borckmans, A. de Wit, B. Rudowics, J.-J. Perraud, E. Dulos, J. Boissonade and P. De Kepper (1995). Pattern selection and localized structures in reaction-diffusion systems. Physica A 213, 181198.

[4] J. Dumais and L. G. Harrison (2000). Whorl morphogenisis in the dasycladalean algae: the pattern formation viewpoint. Phil. Trans. R. Soc. Lond. B 355, 281-305.

[5] M. Field, M. Golubitsky and I. Stewart (1991). Bifurcations on hemispheres. J. Nonlinear Science 1, 201-223.

[6] M. Golubitsky and D. G. Schaeffer (1985). Singularities and Groups in Bifurcation Theory, Vol. I, New York: Springer.

[7] M. Golubitsky, I. Stewart and D. G. Schaeffer (1988). Singularities and Groups in Bifurcation Theory, Vol. II, New York: Springer.

[8] B. C. Goodwin and L. E. H. Trainor (1985). Tip and whorl morphogenesis in Acetabularia by calcium-regulated strain fields. J. Theor. Biol. 117, 79-106.

[9] P. B. Green, C. S. Steele and S. C. Rennich (1996). Phyllotactic patterns: A biophysical mechanism for their origin. Annals of Botany 77, 515-527.

[10] L. G. Harrison (1993). Kinetic Theory of Living Pattern, Cambridge: Cambridge University Press.

[11] L. G. Harrison and M. Kolár (1988). Coupling between reaction-diffusion prepattern and expressed morphogenesis, applied to desmids and dasyclads. J. Theor. Biol. 130, 493-515.

[12] L. G. Harrison and T. C. Lacalli (1978). Hyperchirality: a mathematically convenient and biochemically possible model for the kinetics of morphogenesis. Proc. R. Soc. Lond. B 202, 361-397. 
[13] L. G. Harrison, S. Wehner and D. M. Holloway (2001). Complex morphogenesis of surfaces: theory and experiment on coupling of reactiondiffusion patterning to growth, in Nonlinear Chemical Kinetics: Complex Dynamics and Spatiotemporal Patterns, Faraday Discuss. 120, Royal Society of Chemistry, pp. 277-294.

[14] D. M. Holloway and L. G. Harrison (1995). Order and localization in reaction-diffusion pattern. Physica A 222, 210-233.

[15] D. M. Holloway and L. G. Harrison (1999). Algal morphogenisis: modelling interspecific variation in Micrasterias with reaction-diffusion patterned catalysis of cell surface growth. Phil. Trans. R. Soc. Lond. B 354, 417-433.

[16] A. Hunding and G. D. Billing (1981). Spontaneous pattern formation in spherical nonlinear reaction-diffusion systems: Selection rules favor the bipolar "mitosis" pattern. J. Chemical Physics 75, 486-488.

[17] A. Hunding and M. Brøns (1990). Bifurcation in a spherical reactiondiffusion system with imposed gradient, Physica D 44, 285-302.

[18] T. C. Lacalli (1981). Dissipative structures and morphogenetic pattern in unicellular algae. Phil. Trans. R. Soc. Lond. B 294, 347-388.

[19] T. C. Lacalli (1990). Modelling the Drosophila pair-rule pattern by reaction-diffusion gap input and pattern control in a 4-morphogen system. J. Theor. Biol. 144, 171-194.

[20] M. J. Lyons and L. G. Harrison (1991). A class of reaction-diffusion mechanisms which preferentially select striped patterns. Chem. Phys. Lett. 183, 158-164.

[21] G. Meinhardt (1982). Models of Biological Pattern Formation, London: Academic Press.

[22] G. Meinhardt (1995). The Algorithmic Beauty of Sea Shells, New York: Springer.

[23] G. Nicolis and I. Prigogine (1977). Self-organization in Non-equilibrium Systems, New York: Wiley. 
[24] I. Prigogine and R. Lefever (1968). Symmetry-breaking instabilities in dissipative systems. II. J. Chem. Phys. 48, 1695-1700.

[25] D. Ruelle (1973). Bifurcations in the presence of a symmetry group. Arch. Rational Mech. Anal. 51, 136-152.

[26] A. M. Turing (1952). The chemical basis of morphogenesis. Phil. Trans. R. Soc. Lond. B 237, 37-72.

[27] J. Verdasca, A. de Wit, G. Dewel and P. Borckmans (1992). Reentrant hexagonal Turing structures. Physics Letters A 168, 194-198.

[28] P. von Aderkas (2000). The influence of exogenously applied cytokinin and abscisic acid on cotyledon number of somatic embryos of hybrid larch (Pinaceae), submitted.

[29] W. Zimmermann (1952). Main results of the telome theory. Palaeobotanist 1, 456-470. 